\title{
1 Correlation scan: identifying genomic regions that affect genetic correlations applied to 2 fertility traits
}

3 Babatunde S. Olasege ${ }^{1,2}$, Laercio R. Porto-Neto ${ }^{2}$, Muhammad S. Tahir ${ }^{1,2}$, Gabriela C. 4 Gouveia $^{3}$, Angela Cánovas ${ }^{5}$, Ben J. Hayes ${ }^{4}$ and Marina R. S. Fortes ${ }^{1,4}$

$5{ }^{1}$ The University of Queensland, School of Chemistry and Molecular Biosciences, Saint Lucia Campus, Brisbane, QLD,

6 4072, Australia

$7 \quad{ }^{2}$ CSIRO Agriculture and Food, Saint Lucia, QLD, 4067, Australia

$8{ }^{3}$ Animal Science Department, Veterinary School, Federal University of Minas Gerais, Belo Horizonte 31270-901, Brazil

$9{ }^{4}$ The University of Queensland, Queensland Alliance for Agriculture and Food Innovation (QAAFI), Saint Lucia Campus,

10 Brisbane, QLD, 4072, Australia

$11{ }^{5}$ University of Guelph, Department of Animal Biosciences, Centre for Genetic Improvement of Livestock, 50 Stone Rd E,

12 Guelph N1G 2W1, ONT, Canada

13 *Corresponding author: m.fortes@uq.edu.au

\section{Abstract}

16 Reproductive traits are often genetically correlated. Yet, we don't fully understand the 17 complexities, synergism, or trade-offs between male and female fertility. Here, we introduce 18 correlation scan, a novel framework for identifying the drivers or antagonizers of the genetic 19 correlation between male and female fertility traits across the bovine genome. The 20 identification of these regions facilitates the understanding of the complexity of these traits.

21 Although the methodology was applied to cattle phenotypes, using high-density SNP 22 genotypes, the general framework developed can be applied to any species or traits, and it can 23 easily accommodate genome sequence data.

24 Keywords: Genomic correlation, drivers, antagonizing, RHOGDI, pathway analysis, QTLs, 25 Correlation scan

\section{Background}

28 In animal genetics, insight into the nature of the genetic relationships between quantitative 29 traits are important because they improve our understanding of complex traits and diseases 
$30[1,2]$. These relationships termed genetic correlations manifest when there is shared genetic

31 influence between traits (i.e., pleiotropy) [3, 4] or when there is non-random association

32 between loci (i.e., linkage disequilibrium (LD)) [5, 6]. Estimated genetic correlations provide

33 information on how genome-wide genetic effects align between two complex traits [7].

34 Understanding the interplay between the genomic variants and their effects on quantitative

35 traits can yield insights to improve the prediction of genetic merit and the understanding of

36 complex traits' biology [8-10]. Estimated genetic correlations have informed animal and crop

37 breeding for many decades. For example, scrotal circumference is used as an indicator trait in

38 beef cattle breeding because it is genetically correlated with female fertility traits [11].

39 Nevertheless, we still have a limited information of the regions across genome regulating the

40 intersexual correlations between male and female fertility traits. Investigating these regions

41 and leveraging on the resulting biological information could inspire new approaches in

42 livestock breeding $[12,13]$.

43 Over the past 100 years, different methods have been employed to estimate the genetic

44 correlation between traits [14-17]. Traditionally, these correlations are estimated from

45 pedigree data. However, genome-wide single nucleotide polymorphisms (SNPs) are often

46 used in recent times [18]. It is possible to estimate across-sex correlation between traits and

47 this research niche continues to attract interest among quantitative geneticists [19-21]. The

48 resulting estimates from both within and across-sex analyses range from -1 to +1 , indicating

49 the strength and magnitude of the correlation between traits [22]. Despite more than a century

50 of research on estimating this parameter, it is only very recently that studies attempt to

51 identify the region(s) in the genome that underpin genetic correlations between traits [23-25].

52 In theory, we propose that various genomic regions will contribute to the overall genetic

53 correlation between complex traits. Further, some regions will be driving the genetic 
54 correlation while others might antagonize it. For instance, if the genetic correlation between

55 two traits is 0.70 , some regions will yield a significant and positive correlation, say 0.90,

56 while other regions may antagonize the overall estimate, and in that region the correlation

57 could be -0.50 . Also, some genomic regions may be neutral, say 0.02 and not significant for

58 the correlation between the studied traits. Identifying driver and antagonizing regions are of

59 particular interest if they are for two important traits which are unfavourably correlated, for

60 example milk yield and fertility in dairy cattle. Identification of such regions could lead to

61 more targeted genomic selection and rapid genetic gains for both traits. Current genomic

62 tools have created a great opportunity to advance our knowledge of genetic correlations

63 between complex traits, by investigating the regions in the genome that drive or antagonize

64 these correlations.

65 Here, we introduce a framework termed "correlation scan", which uses a sliding window

66 methodology to uncover the genomic regions driving and antagonizing genetic correlations in

67 beef cattle. We applied the method to male and female fertility traits and showcase how the

68 outcomes of this methodology can be interpreted in downstream analyses to gain further

69 insight about the studied traits and their relationships. Reproductive traits are often

70 genetically correlated, and yet we don't fully understand the complexities, synergism, or

71 trade-offs between male and female fertility. To demonstrate the method, we used two pairs

72 of reproductive traits with strong genetic correlations in two independent cattle populations

73 from our previous study [26]. These traits are age at first corpus luteum (AGECL, i.e., female

74 puberty) and serum levels of insulin growth hormone (IGF1 measured in bulls, IGF1b, or

75 cows, IGF1c). These pairs of traits serve as example of a positive and a negative correlation

76 between phenotypes measured in males and females, during pubertal development. The

77 populations used in the study are formed by either Brahman (BB) cattle or Tropical

78 Composite (TC) cattle, as described in our previous study [26]. 


\section{Results}

\section{The total number of windows generated and analysed}

81 Using the framework developed in our study (see Materials and Methods), genomic windows

82 with their corresponding correlation estimates (r) for each pairwise trait in two beef cattle

83 populations were identified. The total number of windows generated for all pairwise traits in

$84 \mathrm{BB}$ was 5,558 and the number in TC was 6,876. For all windows, the chromosome

85 coordinates and the corresponding $\mathbf{r}$ estimates in each of the two populations are presented in

86 Additional file 1 (Table S1-S2). The $\mathbf{r}$ estimates for all windows were plotted against their

87 genomic position (i.e., midpoint between the start and end position of each window) (Figure

88 1). Results are presented separately per cattle population and for each pair of traits

89 investigated 


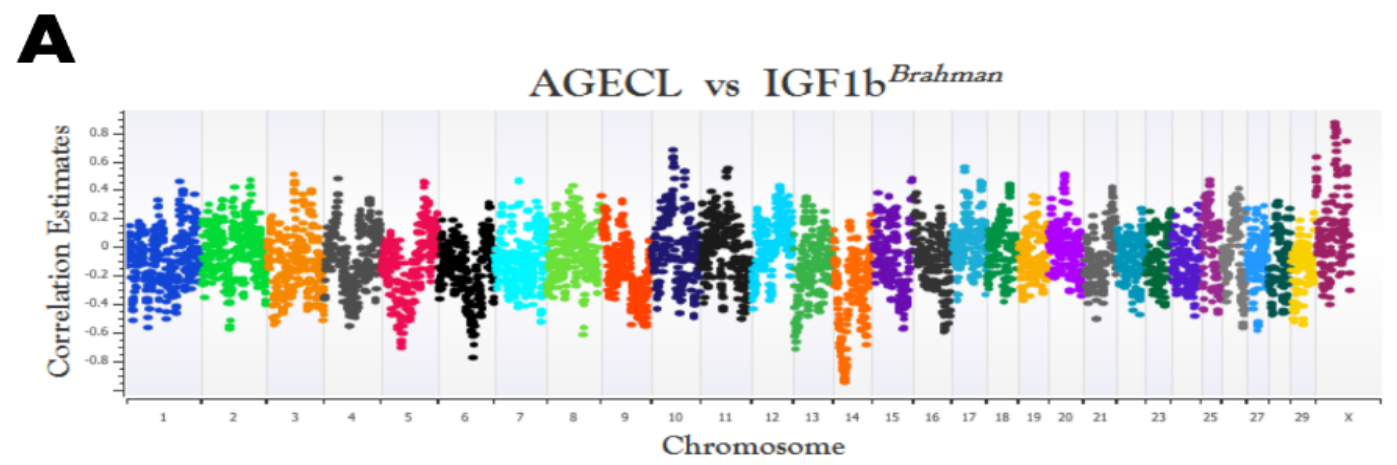

B

AGECL vs IGF1B Tropical Composite $^{\text {Th }}$

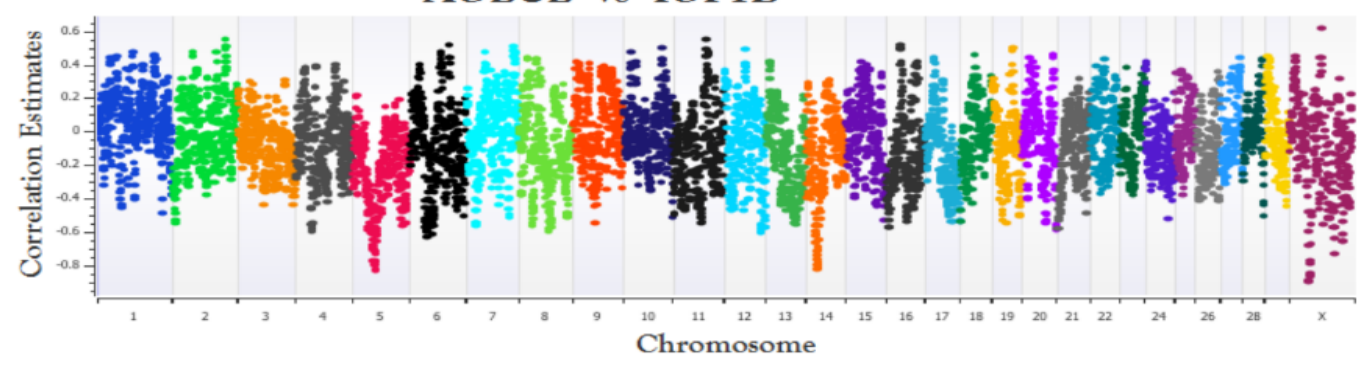

C

IGF1c vs IGF1b $\mathrm{b}^{\text {Brahman }}$

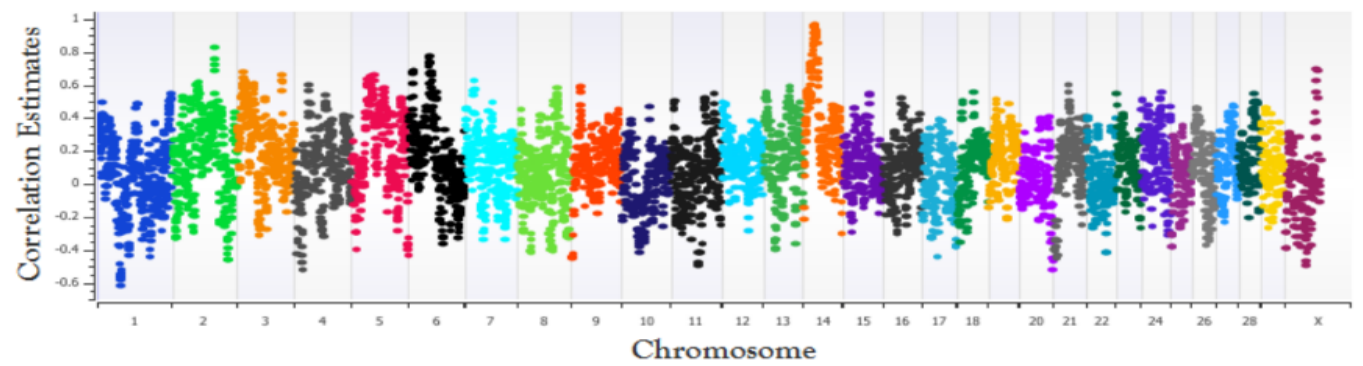

D

IGF1c vs IGF1b Tropical Composite

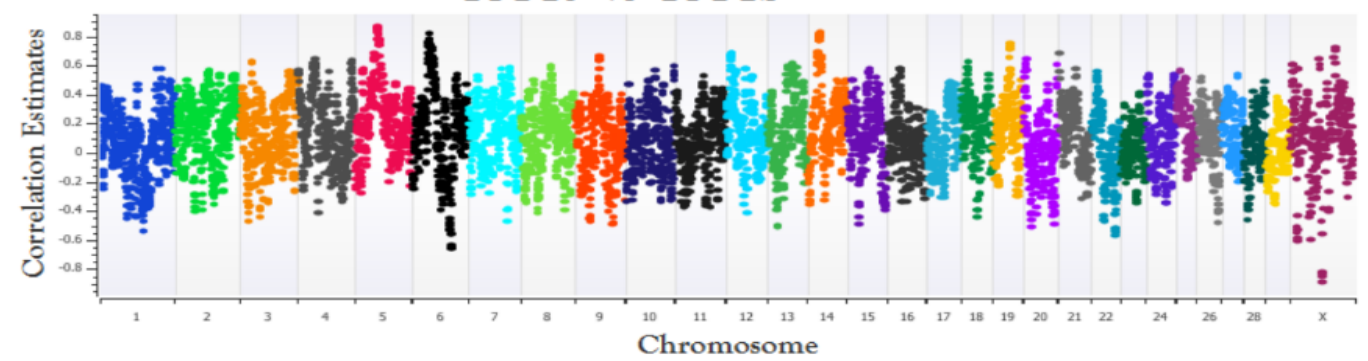

90

Figure 1. Genome plot of the regions driving and antagonizing trait correlations in Brahman (BB) and Tropical Composite (TC) for the pairwise traits (BB-AGECL vs IGF1b; A, TCAGECL vs IGF1b; B, BB-IGF1c vs IGF1b; C, TC- IGF1c vs IGF1b; D). AGECL, age at first corpus; IGF1, serum levels of insulin growth hormone (measured in bulls, IGF1b, or cows, IGF1c). The correlation estimates were plotted on the y-axis and the genomic position (i.e., midpoint between the start and end position of each window) of each chromosome on the $\mathrm{x}$-axis, according to the ARS_UCD1.2 bovine reference genome. 
98 Driver, antagonizing and neutral genomic windows affecting genetic correlations between fertility traits: permutation test

In order to identify drivers, antagonizing and neutral regions across the bovine genome, we performed permutation test by randomly reshuffling the Single Nucleotide Polymorphisms (SNPs) effects in each window across all chromosomes in 1,000 iterations for each trait. Then, we applied our framework on the randomized SNP effects and observed the $\mathbf{r}$ estimate across each iteration for each window. In most cases, the maximum and the minimum $\mathbf{r}$ estimates for each window at each iteration (i.e., rand 1 to rand 1,000) range between \pm 0.20 . Therefore, we considered neutral windows with no significant effect on the trait correlation as windows with $-0.20 \leq \mathbf{r} \leq 0.20$ estimates. The genomic plots of the $\mathbf{r}$ estimates resulting from the permutation test (rand 500 only) in each population are presented in Additional file 2 (Fig. S1). Additional file 3 (Table S3-S6) shows the numbers of windows, their chromosome coordinates, and $\mathbf{r}$ estimates as well as the maximum and minimum $\mathrm{r}$ estimates across the 1,000 iterations for each pair of traits in each of the two populations.

As a result of the permutation test, we considered significant windows with $\mathbf{r}$ estimates $>0.2$ and $\mathbf{r}$ estimates $<-0.2$. These thresholds were used to define the significant windows or regions (i.e., driver and antagonizing) from non-significant (i.e., neutral) windows or regions. Depending on the overall genetic correlation between traits, driver and antagonizing windows can be deduced: in driver windows, the $\mathbf{r}$ estimate has the same direction, positive or negative, as the overall genetic correlation; in antagonizing windows it is the opposite.

The number of significant driver windows for the correlation between AGECL and IGF1b was 1,636 in $\mathrm{BB}$ and 1,914 in $\mathrm{TC}$ cattle. The number of significant windows for the antagonizing was 547 in BB and 898 in TC cattle, for AGECL vs IGF1b. For the correlation between IGF1c and IGF1b, the number of significant driver windows was 1,931 in BB and 
122 2,549 in TC cattle. The antagonizing windows was 402 in BB and 587 in TC cattle (IGF1c vs

123 IGF1b). The numbers of neutral windows were as follows: 3,375 in BB, and 4,064 in TC for

124 AGECL vs IGF1b; and 3,225 in BB, and 3,740 in TC for IGF1c vs IGF1b. See Table 1 for

125 details on numbers of windows in the two beef cattle populations. In addition, the lists of

126 windows with their chromosomal coordinates for all driver, antagonizing, and neutral regions

127 are presented in Additional file 4 (Table S7-S18).

128 Table 1: The number of windows generated for the driver, antagonizing and neutral 129 windows for each pairwise trait in Brahman and Tropical Composite population.

\begin{tabular}{|c|c|c|c|c|}
\hline \multirow[t]{3}{*}{ Pairwise Trait } & \multicolumn{3}{|c|}{ Number of windows (percentage to the total number of } & \multirow{3}{*}{$\begin{array}{l}\text { Total number } \\
\text { of windows }\end{array}$} \\
\hline & \multicolumn{3}{|c|}{ window) } & \\
\hline & Driver $(\%)$ & Antagonizing (\%) & Neutral (\%) & \\
\hline \multicolumn{5}{|c|}{ Brahman } \\
\hline AGECL vs IGF1b & $1,636(29.40 \%)$ & $547(9.84 \%)$ & $3,375(60.72 \%)$ & 5,558 \\
\hline IGF1c vs IGF1b & $1,931(34.74 \%)$ & $402(7.23 \%)$ & $3,225(58.03 \%)$ & 5,558 \\
\hline \multicolumn{5}{|c|}{ Tropical Composite } \\
\hline AGECL vs IGF1b & $1914(27.84 \%)$ & $898(13.06 \%)$ & $4064(59.10 \%)$ & 6,876 \\
\hline IGF1c vs IGF1b & $2549(37.73 \%)$ & $587(8.54 \%)$ & $3740(54.39 \%)$ & 6,876 \\
\hline
\end{tabular}

130 AGECL, age at first corpus; IGF1c, serum levels of insulin growth hormone measured in 131 cow; IGF1b, serum levels of insulin growth hormone measured in bulls

132

133 For the correlation between AGECL and IGF1b (overall genome-wide correlation of -0.65

134 (BB) and -0.55 (TC), see Table 2), the largest $\mathbf{r}$ estimate for the driver windows was -0.96

135 (bovine chromosome (BTA)14: $23.04-25.29 \mathrm{Mb}$ ) in BB and -0.91 (BTAX: $39.76-42.86 \mathrm{Mb})$ 
136 in TC. For the antagonizing windows, the largest $\mathbf{r}$ estimate was 0.87 (BTAX: 40.87 -

$13743.88 \mathrm{Mb}$ ) in BB and 0.61 (BTAX: $66.62-69.622 \mathrm{Mb})$ in TC.

138 For the correlation between IGF1c and IGF1b (overall genome-wide correlation of 0.86 (BB)

139 and 0.93 (TC), see Table 2), the largest estimate for the driver windows was 0.97 (BTA14:

$14022.68-24.96 \mathrm{Mb})$ in $\mathrm{BB}$ and 0.87 (BTA5: 46.13- 47.89Mb) in TC, while the estimate for the

antagonizing was -0.62 (BTA1: $49.01-51.67 \mathrm{Mb})$ in BB and -0.90 (BTAX: $65.64-68.39 \mathrm{Mb})$

in TC. All $\mathbf{r}$ estimates are plotted in Figure 1.

Genes and quantitative trait loci (QTL) within driver and antagonizing regions across

\section{the two populations}

Defining driver and antagonizing regions separately for each pair of traits, allowed us to identify the genes and QTLs within these regions for each of the two beef cattle populations.

The percentage of the overlapping genes (Figure 2) and QTLs (Figure 3) across both populations was studied. The percentages of genes shared across the significant regions in BB and TC were calculated as a function of the total number of genes in BB or TC, respectively, and so they differ (Figure 2 and 3).

The percentage of overlapping genes for each pair of traits in the two populations were as follows: for AGECL vs IGF1b driver regions, about $48 \%$ of the total number of genes annotated were shared between the two population, whereas, for the antagonizing regions, $22 \%$ of the gene annotated in BB were present in the TC population, and $26 \%$ of the genes annotated in TC were present in BB; for IGF1c vs IGF1b, the two populations shared about $58 \%$ of total number of genes annotated for the driver regions and about $17 \%$ were shared for the antagonizing regions. 
160 present in TC and 35\% of the QTLs annotated in TC were present in BB, whereas, for the

161 antagonizing regions, $20 \%$ of the QTLs annotated in BB were present in TC and $18 \%$ of the

162 QTLs annotated in TC were present in BB; for IGF1c vs IGF1b, 56\% of the genes annotated

163 in $\mathrm{BB}$ were present in $\mathrm{TC}$, and $52 \%$ of the genes annotated in $\mathrm{TC}$ were present in $\mathrm{BB}$,

164 whereas, for the antagonizing regions, $29 \%$ of the genes annotated in BB were present in TC

165 and $33 \%$ of the genes annotated in TC were present in BB population. 


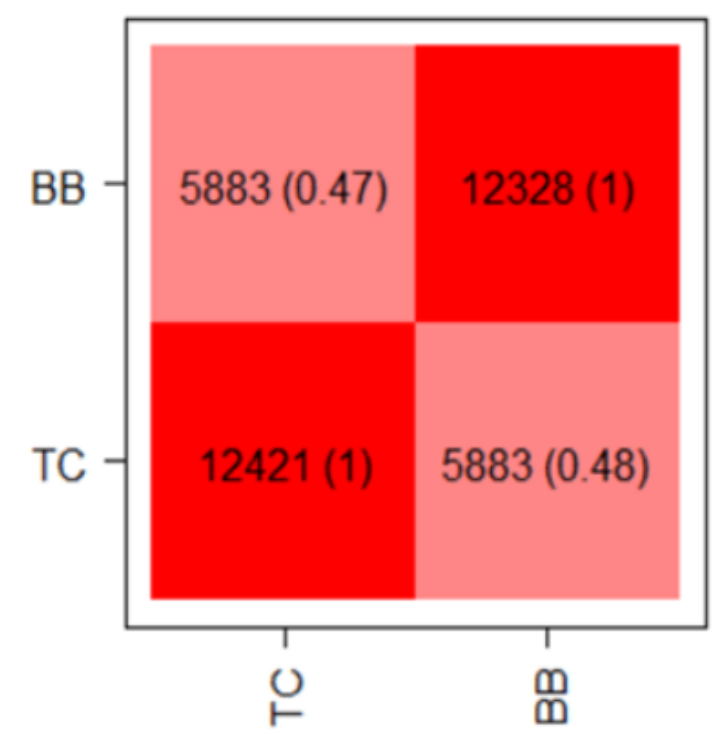

AGECL vs IGF1b

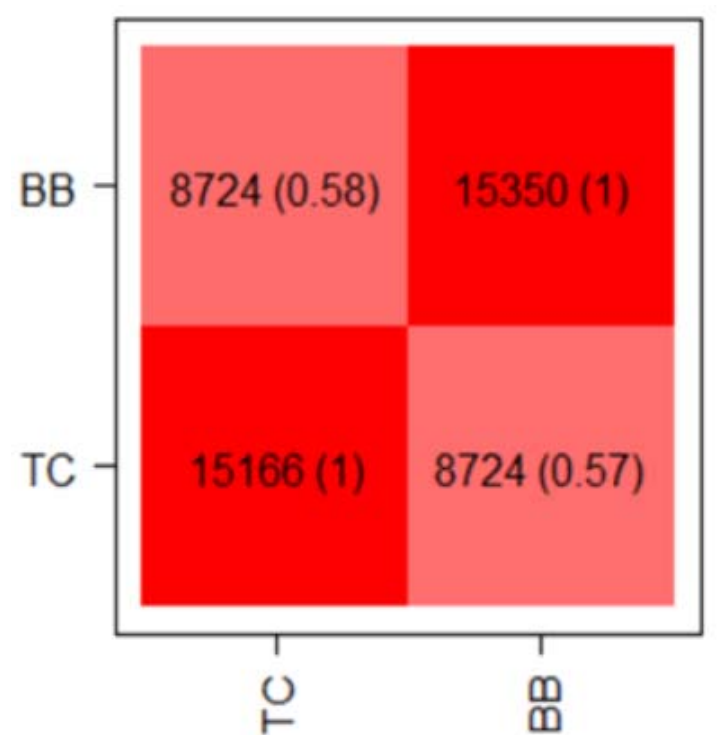

IGF1c vs IGF1b

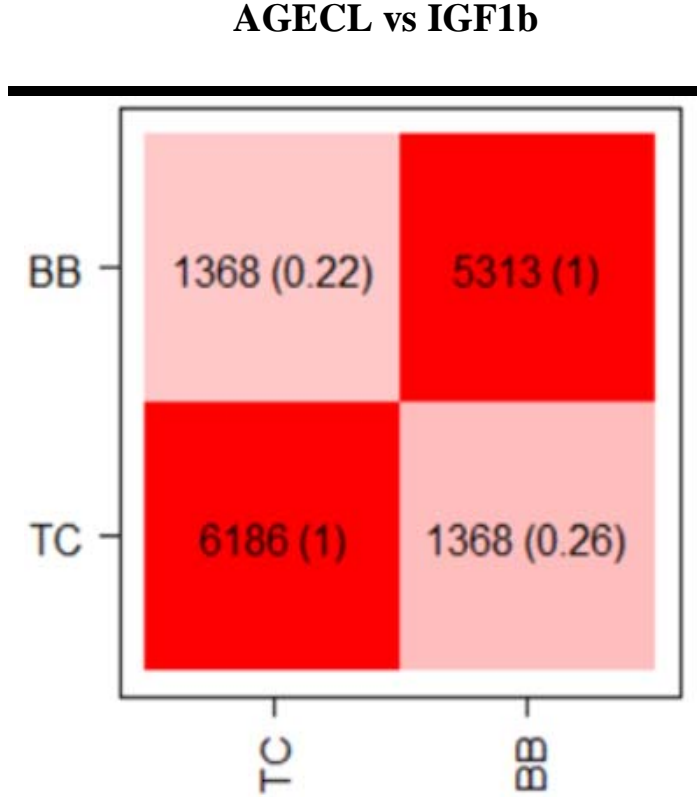

AGECL vs IGF1b

\section{Antagonizing}

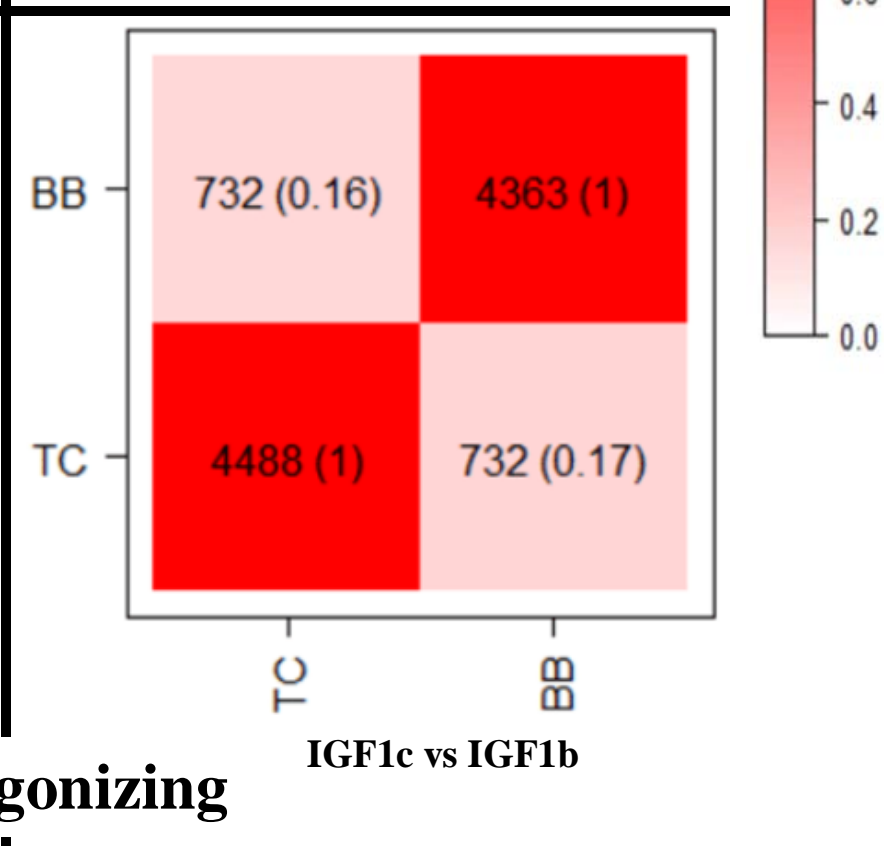

Figure 2. Genes annotated in the significant (i.e., driver and antagonizing) genomic regions identified as explaining the genetic correlations between male and female fertility traits in Brahman (BB) and Tropical Composite (TC) population. The overlaps between the two studied populations are in the diagonal of each plot for each pair of traits within the driver (above) and antagonizing (below) regions. The darker the colour within the squares, the higher the percentage of shared genes or QTLs. AGECL, age at first corpus; IGF1, serum levels of insulin growth hormone (measured in bulls, IGF1b, or cows, IGF1c). 


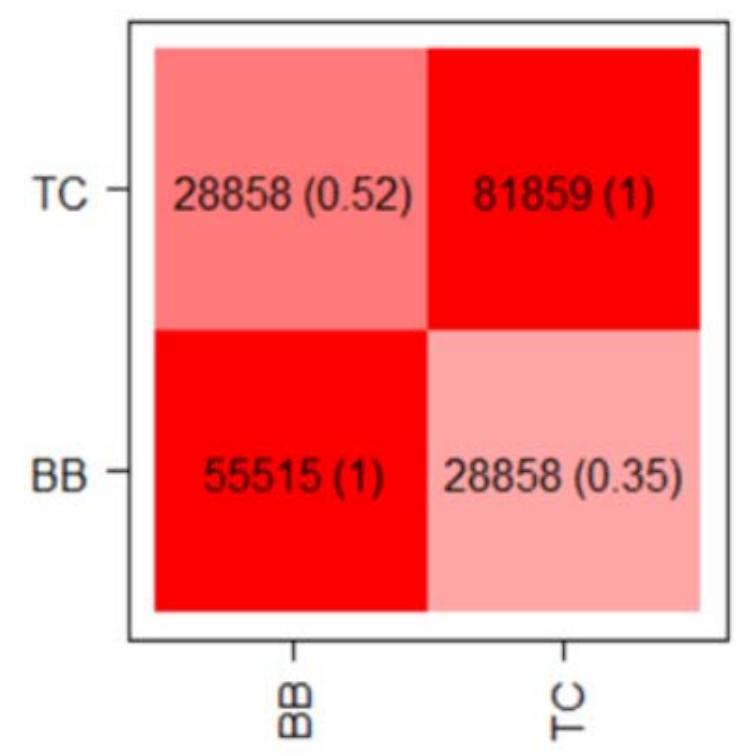

AGECL vs IGF1b

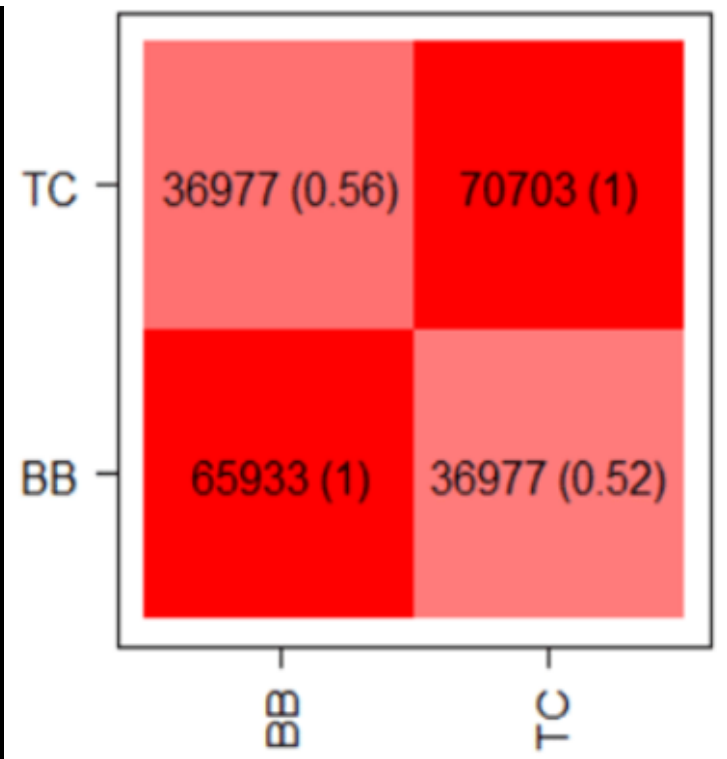

IGF1c vs IGF1b

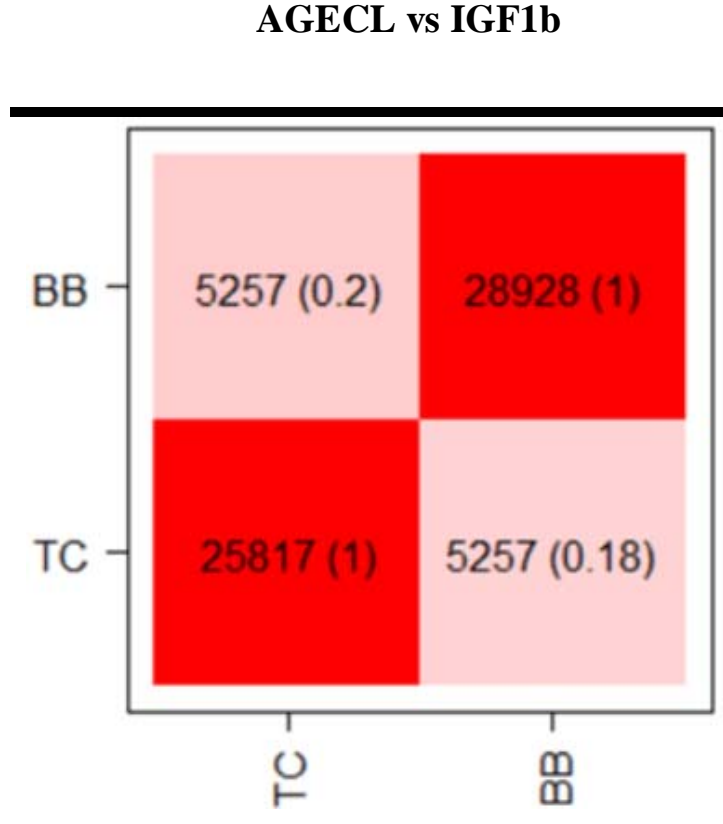

AGECL vs IGF1b

\section{Antagonizing}

Figure 3. QTLs annotated in the significant (i.e., driver and antagonizing) genomic regions identified as explaining the genetic correlations between male and female fertility traits in Brahman (BB) and Tropical Composite (TC) population. The overlaps between the two studied populations are in the diagonal of each plot for each pair of traits within the driver (above) and antagonizing (below) regions. The darker the colour within the squares, the higher the percentage of shared genes or QTLs. AGECL, age at first corpus luteum; IGF1, serum levels of insulin growth hormone (measured in bulls, IGF1b, or cows, IGF1c). 
184 Functional classification of QTLs within genomic regions that explain the genetic correlations between male and female fertility

186 To infer biological function and mine the existing literature, we examined the types of QTL

187 (milk, reproduction, production, meat and carcass, health and exterior) present in the 188 significant genomic regions identified above using GALLO [27]. The most frequent QTLs 189 across all pairwise traits in the two populations for the driver and antagonizing regions were 190 QTLs related to milk production, accounting for about $30-51 \%$ in most cases. This was 191 followed by reproductive QTLs accounting for about 13-48\% and production QTLs 192 comprising 6-24\%. Other QTL types (Exterior, health and meat and carcass) accounted for a 193 relatively small proportion of QTLs in the significant regions (Figure 4 and 5). In addition, 194 we report the top 10 results for QTLs related to reproductive traits as these are relevant to our 195 studied traits (Figure 4 and 5). Among these reproductive QTLs, traits related to puberty (i.e., 196 age at puberty, scrotal circumference) were prevalent in both populations. 
bioRxiv preprint doi: https://doi.org/10.1101/2021.11.05.467409; this version posted January 10, 2022. The copyright holder for this preprint (which was not certified by peer review) is the author/funder, who has granted bioRxiv a license to display the preprint in perpetuity. It is made available under aCC-BY 4.0 International license.

197

198

\section{BB}

\section{A}

" Exterior

Health

Meat and Carcass

= Milk

" Production

" Reproduction

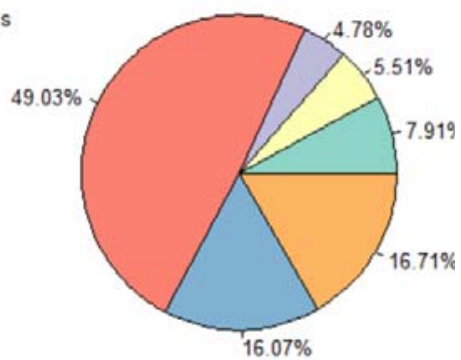

Exterior

Health

" Meat and Carcass

" Milk

= Production

" Reproduction

TC

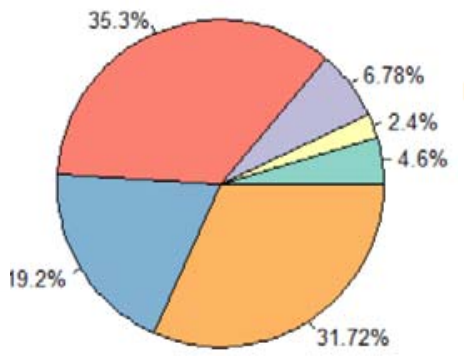

\section{AGECL-IGF1b-Driver}
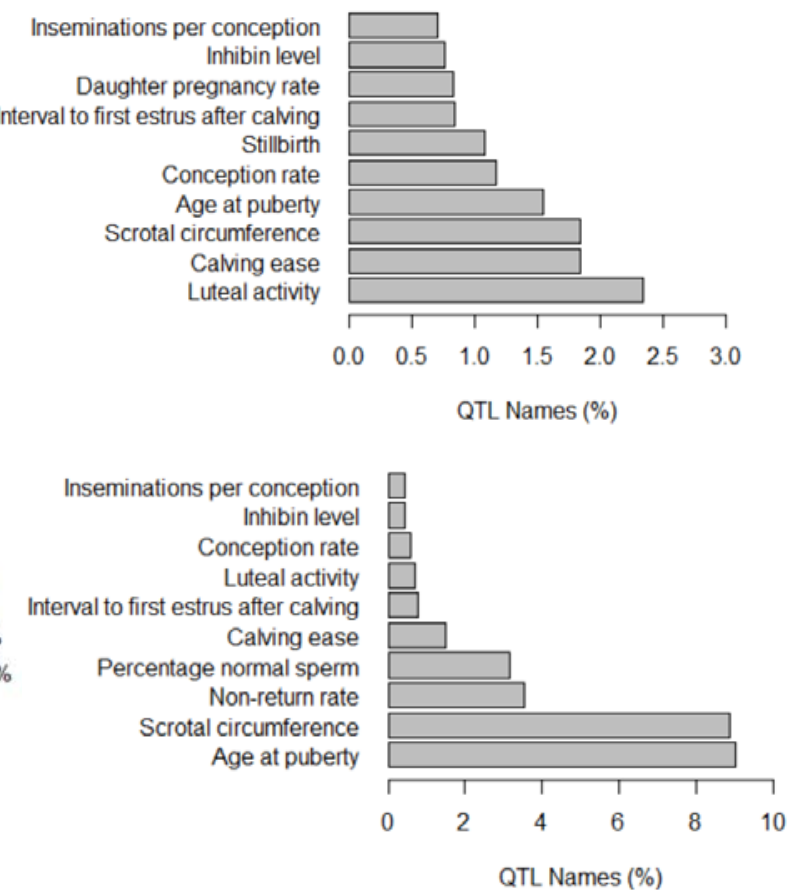

199

AGECL-IGF1b-Antagonizing

200

\section{B}

= Exterior
Health
$=$ Meat and Carcass
$=$ Milk
$=$ Production
$=$ Reproduction

BB

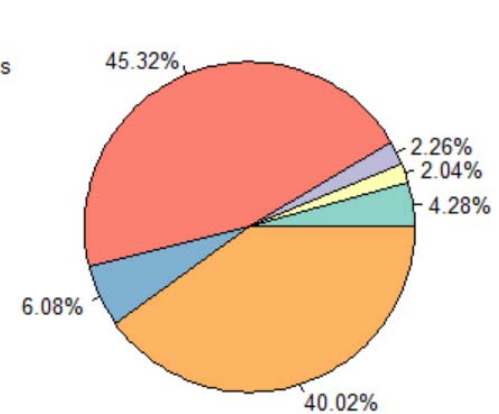

201

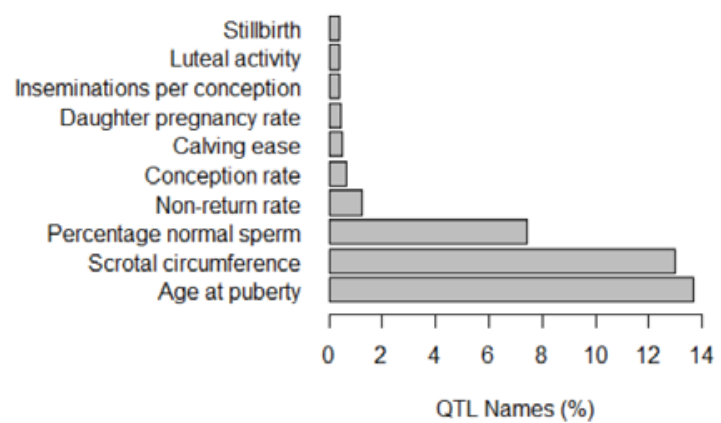

QTL Names (\%)

\footnotetext{
- Exterior

Health

= Meat and Carcass

" Milk

- Production

" Reproduction
}
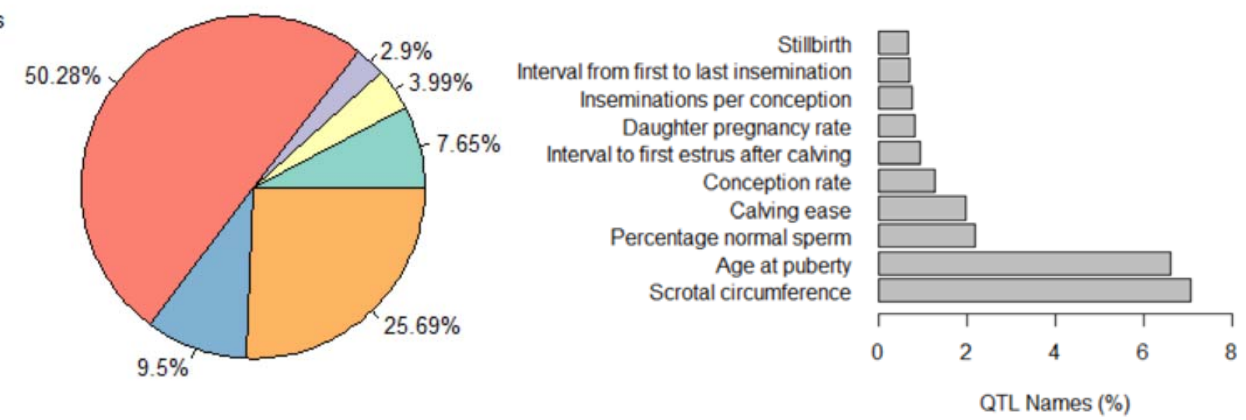

Figure 4. Percentage of QTL type (pie chart) and trait related to reproduction QTLs

204 (barplots) for the QTL annotation results obtained for (A) AGECL vs IGF1b - driver, (B)

205 AGECL vs IGF1b- antagonizing in Brahman (BB) and Tropical Composite (TC) population.

206 AGECL, age at first corpus luteum, IGF1, serum levels of insulin growth hormone

in bulls,

IGF1b,

or

cows,

IGF1c) 
bioRxiv preprint doi: https://doi.org/10.1101/2021.11.05.467409; this version posted January 10,2022 . The copyright holder for this preprint (which was not certified by peer review) is the author/funder, who has granted bioRxiv a license to display the preprint in perpetuity. It is made available under aCC-BY 4.0 International license.

\section{8}
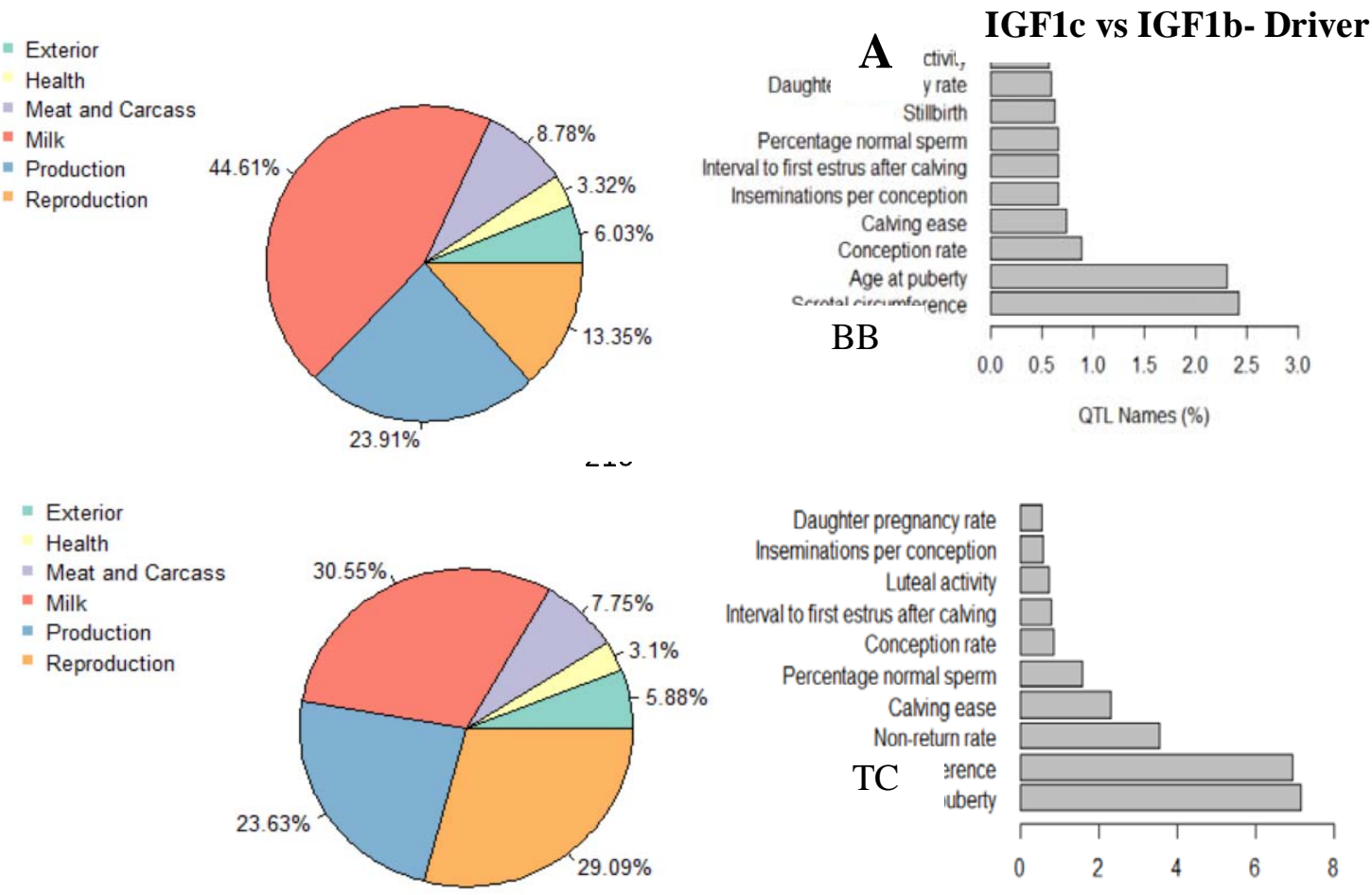

IGF1c vs IGF1b-Antagonizing

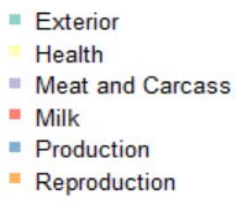

= Exterior Health

" Meat and Carcass

- Milk

- Production

" Reproduction

TC IGF1c).

\section{B
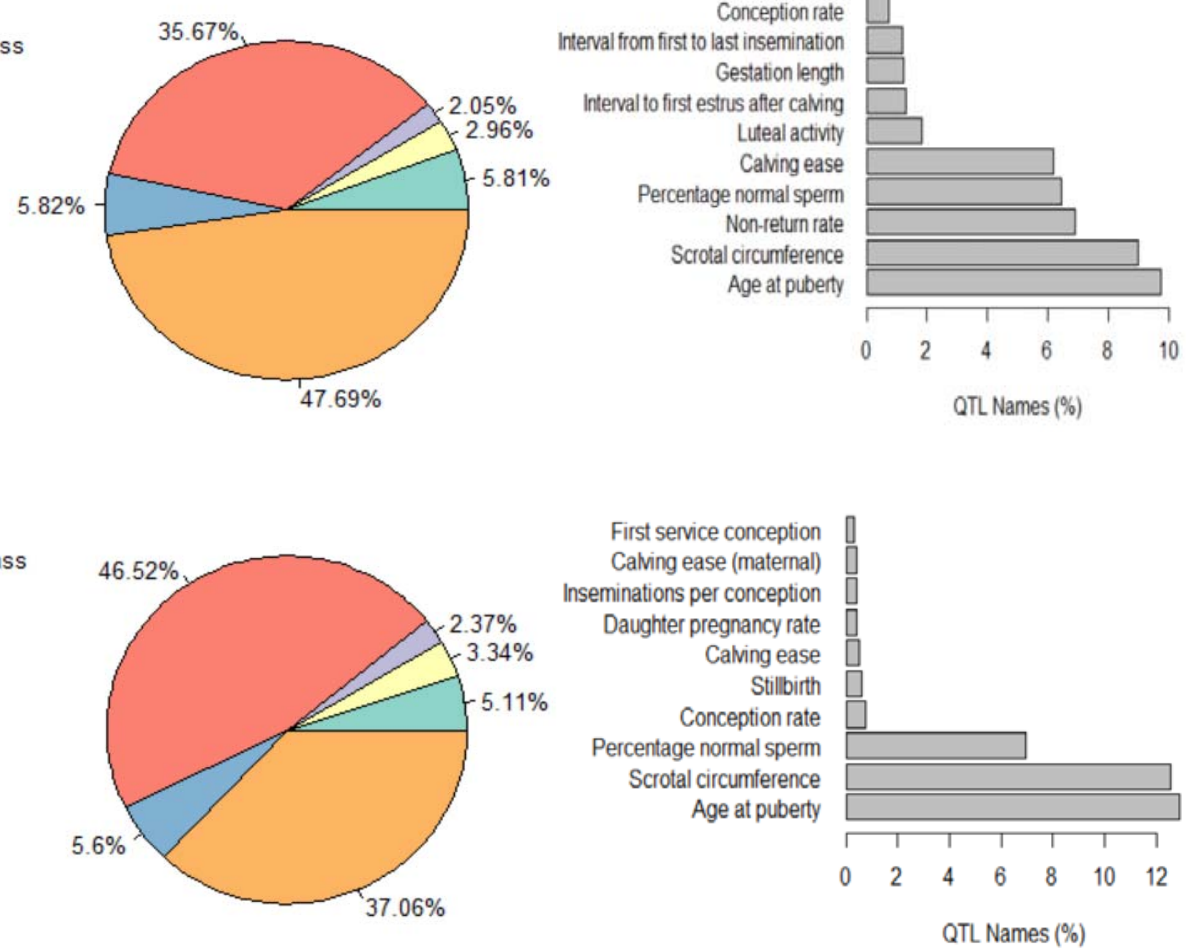

Figure 5. Percentage of QTL type (pie chart) and trait related to reproduction QTLs (barplots) for the QTL annotation results obtained for (A) IGF1c vs IGF1b- driver, (B) IGF1c vs IGF1b- antagonizing in Brahman (BB) and Tropical Composite (TC). AGECL, age at first corpus luteum, IGF1, serum levels of insulin growth hormone (in bulls, IGF1b, or cows, 


\section{QTL enrichment analysis}

221 We performed a chromosome-wide QTL enrichment analysis to further test the significance

222 of the QTLs identified for all the driver and antagonizing regions in each cattle population,

223 for each trait pair using GALLO [27]. Enriched QTLs for the studied traits span across most

224 QTL types, indicating the presence of complex genetic mechanisms. The results of the

225 chromosome-wide QTLs enrichment (FDR-corrected p-value $\leq 0.05$ ) for the driver and

226 antagonizing regions for all pairwise traits in each population are presented in Additional file

2275 (Table S19-26).

228 For the driver regions, the number of QTLs enriched over a wide range of chromosomes for 229 AGECL vs IGF1b were 233 and 144 in BB and TC beef cattle population, respectively. The 230 number was 227 (BB) and 220 (TC) for IGF1c vs IGF1b. For AGECL vs IGF1b, the most 231 enriched chromosome (no of enriched QTLs in parenthesis) was BTA5 (36) and BTA14 (18)

232 in $\mathrm{BB}$ and $\mathrm{TC}$, respectively. IGF1c vs IGF1b also followed similar pattern with the result 233 above, with BTA5 (41) being the most enriched chromosome in BB and BTA14 (51) as the 234 most in TC.

235 For the antagonizing regions, the number of QTLs enriched across the bovine chromosomes 236 for AGECL vs IGF1b were 127 and 178 in BB and TC beef cattle population, respectively. 237 The number was 179 (BB) and 195 (TC) for IGF1c vs IGF1b. For AGECL vs IGF1b, the 238 most enriched chromosome was BTA17 (14) and BTA26 (21) in BB and TC, respectively. 239 For IGF1c vs IGF1b, however, BTA14 (23) was the most enriched chromosome for these 240 regions in BB, whereas, in TC, BTA14 (23) was the most enriched.

241 To identify the common results and shared biology between the driver and the antagonizing 242 regions, we also investigated the overlaps of the QTL types associated with the studied trait 243 (i.e., reproduction) in the two populations. The relationship between the top 10 enriched 
244 reproductive QTLs in BB and TC are presented in Figure 6. Irrespective of the trait pair, for

245 the driver regions, the reproductive QTLs in BB in most cases overlap with those identified in

246 TC. However, for the antagonizing regions, not all reproductive QTLs in BB were found in

247 TC

beef

cattle

population. 


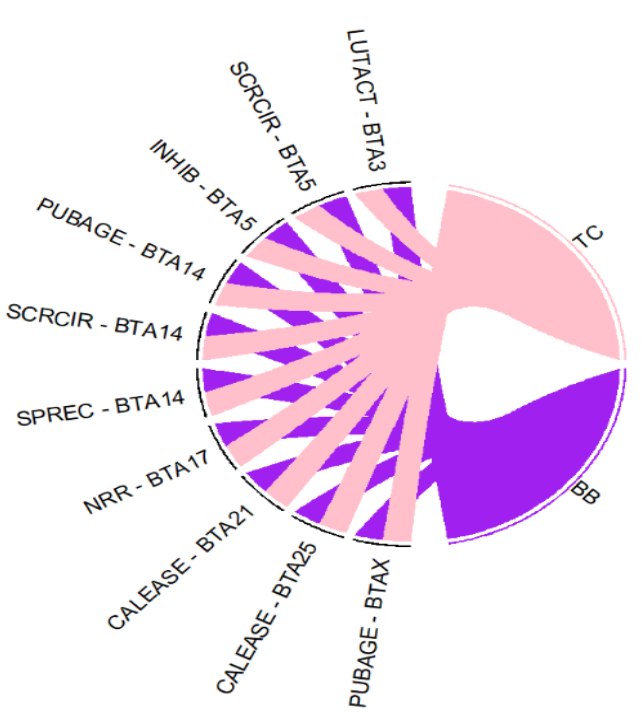

AGECL vs IGF1b

\section{Driver}

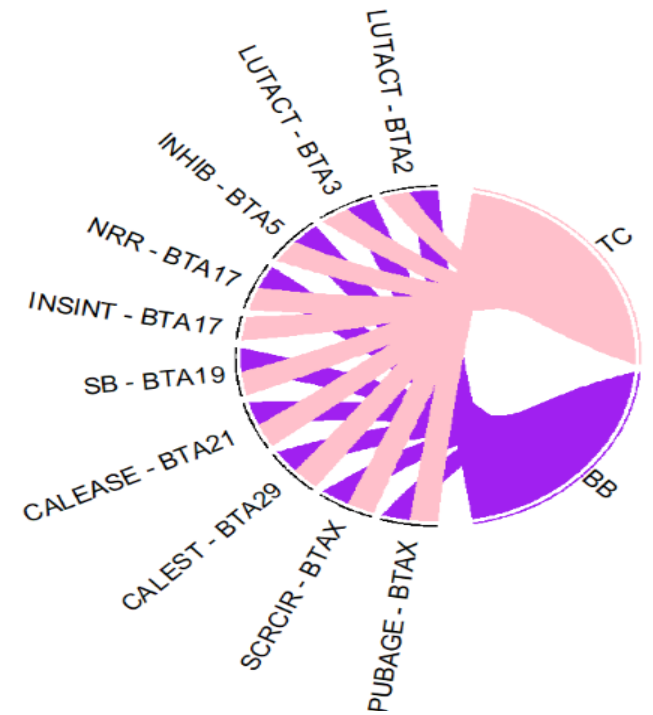

IGF1c vs IGF1b

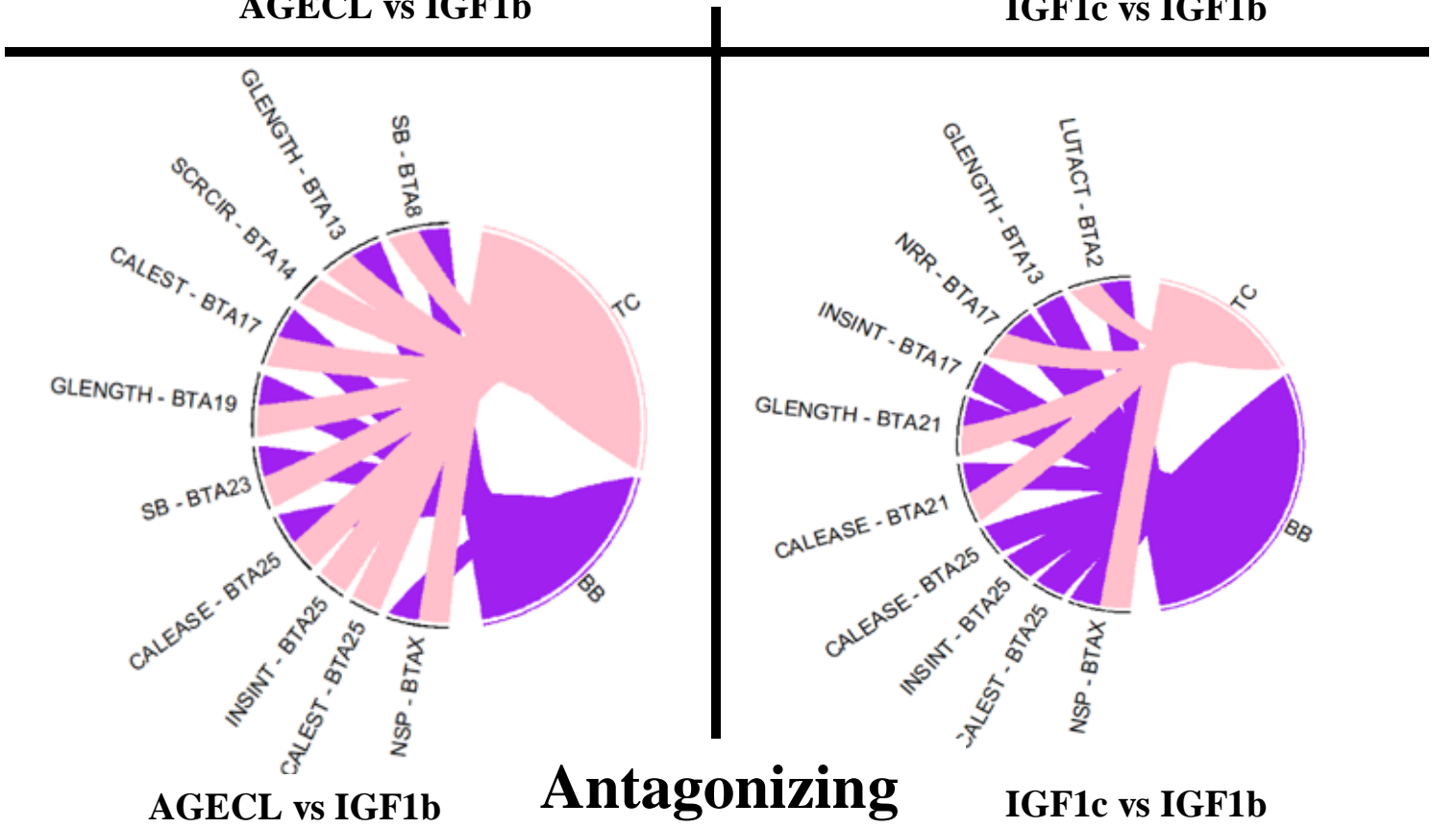

Figure 6. Chord plot showing the relationship between the top 10 enriched reproductive QTLs between Brahman (BB) and Tropical Composite (TC) for the driver (top) and the antagonizing (bottom) regions of the studied traits. AGECL, age at first corpus luteum, IGF1c, serum levels of insulin growth hormone measured in cow; IGF1b. LUTACT, Luteal activity; SCRCIR, Scrotal circumference; INHIB, Inhibin level; PUBAGE, Age at puberty; SPREC, Sexual precocity; NRR, Non-return rate; CALEASE, Calving ease; INSINT, Interval from first to last insemination; SB, Still birth; CALEST, Interval to first estrus after calving; GLENGTH, Gestation length, NSP, Percentage normal sperm. 


\section{$259 \quad$ Functional enrichment analysis}

260 Leveraging our methodology's directionality of gene effects with Ingenuity Pathway 261 Analysis (IPA; http://www.ingenuity.com), we identified the enriched canonical metabolic 262 pathways enriched at Benjamini-Hochberg corrected $\mathrm{p}$-values $(\mathrm{BH}-\mathrm{P}$-value) of $\mathrm{p}<0.01$. The 263 graphical presentation of the canonical metabolic pathways predicted by IPA to be enriched 264 and the proportion of driver and antagonizing genes in each pathway for all pairwise traits 265 investigated in each population are illustrated in Additional file 6 (Fig. S2-S9). Although IPA 266 provided information about whether the predicted pathways were being activated or inhibited 267 based on our data, we remain cautious when interpreting our results since the $\mathbf{r}$ estimates are not the same as gene expression values, and IPA was originally designed to mine gene expression data.

270 The number of pathways enriched for AGECL vs IGF1b was 49 in BB and 68 in TC. For 271 IGF1c vs IGF1b, the number of enriched pathways was 156 and 87 in $\mathrm{BB}$ and TC, 272 respectively. For AGECL vs IGF1b in BB, the top 5 enriched canonical metabolic pathways 273 were cardiac hypertrophy signaling (Enhanced), toll-like Receptor signaling, IL-6 Signaling, 274 hepatic fibrosis signaling pathway and STAT3 Pathway. In TC population, the top 5 enriched 275 canonical metabolic pathways were breast cancer regulation by stathmin1, signaling by Rho 276 family GTPases, opioid signaling pathway, endocannabinoid developing neuron pathway, and CREB signaling in neurons.

For IGF1c vs IGF1b in BB, the top 5 enriched canonical metabolic pathways were cardiac hypertrophy signaling, CREB signaling in neurons, thrombin signaling, estrogen receptor

280 signaling, opioid signaling pathway and AMPK signaling. In TC, the top 5 enriched pathways were CREB signaling in neurons, cardiac hypertrophy signaling (enhanced), opioid signaling pathway, gas signaling and breast cancer regulation by stathmin 1 . The enriched canonical 
283 metabolic pathways and all the genes involved in each pathway are available in Additional

284 file 7 (Table S27-30).

\section{Discussion}

286 Complex phenotypes, including fertility, consist of multiple genetically correlated rather than

287 independent traits [6]. The interplay between traits involve many genomic regions, usually in

288 a large and polygenic regulatory network [28-31]. Genomic signals that regulate (i.e., drive or

289 antagonize) complex traits are widely spread across the genome, including near many genes

290 without significant effect on the phenotype or disease [29]. In the present post-genomic era,

291 unravelling the genomic regions that regulate complex traits and the metabolic pathways

292 associated with these phenotypes has become an important aspect of genetic studies in

293 humans and animals [32]. In this study, we developed a novel framework termed correlation

294 scan to reveal the significant regions that either drives or antagonize the genetic correlations

295 between traits, across the genome. In addition, this method can also reveal genomic regions

296 with no effect on the studied traits (neutral windows). The framework developed uses best

297 linear unbiased prediction (BLUP) solution of SNP effects to estimate the local correlations

298 between studied traits. Local correlations are based on sliding windows of 500-SNPs. We

299 applied these sliding windows approach to reproductive traits measured in two populations

300 and subject the outcomes, significant windows, to further analyses using GALLO [27]. and

301 IPA (http://www.ingenuity.com) to gain further insight about the biology of studied traits and

302 their relationships. Although the methodology was applied to beef cattle traits, using high-

303 density SNP chip genotypes, the general framework can be applied to any species, any traits,

304 and it can easily accommodate sequence level data.

305 Our results agreed with the established notion that multiple loci regulate reproductive traits

306 [33-35]. Also, the mode of action of these loci and the magnitude of their effect varies across 
307 the genome. While some regions had no effect on the genetic correlations under 308 investigation, other loci drive or antagonize the relationships between male and female 309 fertility. The identification of driver and antagonizing loci creates opportunities to further 310 understand the molecular mechanisms affecting quantitative traits. For example, correlations 311 estimated from SNP effects have allowed researchers to construct gene networks [36]. 312 Thereby, these types of approaches contribute to linking genotype with phenotype.

313 The two beef cattle populations investigated in this study are distinct in terms of their genetic 314 composition. Brahman (BB) cattle are typically of Bos indicus origin whereas TC beef cattle 315 emanated from the crossing between Bos indicus and Bos taurus breed [37]. Despite these 316 differences, we found that a considerable number of annotated genes and QTLs driving trait 317 correlation overlaps across breeds, although with variations in the size of SNP effects. This 318 corroborates the findings of Bolormaa, Pryce [38], where a substantial number of QTLs were 319 found segregating in Bos indicus and composite cattle using the same dataset. In this present 320 study, the top genomic signal driving trait correlation across all pairwise traits in $\mathrm{BB}$ were 321 located on BTA14. The significant region contains a widely known and well-characterized 322 QTL, including the PLAG1 gene, reported to be associated with growth and reproductive 323 traits in our populations and other studies [39-44]. In TC however, the top signal differs 324 across traits and mostly spread across two or three chromosomes, although with considerable number of overlaps with BB. This could be partly due to the variations in the architecture of 326 composite breed [45]. The genome of composite breeds usually contains new haplotypes 327 emerging from generations of crossbreeding. Moreover, the contribution of the founder 328 populations on chromosomes and specific genomic regions are usually unevenly distributed, 329 which most likely shapes the genome of composite breeds [45]. In short, differences between $330 \mathrm{BB}$ and TC are likely to impact the results of our analyses. Breed differences are expected, 
and so when two breeds share a similar result, it enhances our confidence in calling significant windows for the interplay between male and female fertility traits.

Most genomic regions antagonizing the genetic correlations between male and female

334 fertility traits were located on chromosome X. Gene expression on chromosome X differs across-sex, resulting in genomic sexual conflict [46-48]. Genes in these antagonizing regions include $P O 1 F B, Z N F 711, A P O O L, H D X, D A C H 2, F A M 133 A$, among others. These genes are associated with different disorders including infertility, reproductive deficiencies, primary ovarian failure [49-51]. When some of these genes are over-expressed, it can dysregulate the cristae morphology of the mammalian mitochondria [52]. Understanding how these antagonizing genes interact to influence (in)fertility could help improve the reproductive potentials of beef cattle.

In animal production, more research is carried out on milk production-related traits, thereby creating large proportion of records for these traits in the cattle QTL database. These volumes of records can create a bias in the QTLs representativeness [27]. The QTL enrichment analysis allows testing the significance of the QTL representative using chromosome-wide approach to detect specific genomic region with many QTLs for a specific trait. For example, taking the driver regions for AGECL vs IGF1b in BB, the top enriched QTLs was found in BTA5, harbouring 36 QTLs. These QTLs comprised 8 different QTLs for reproduction (inhibin level, scrotal circumference, interval of first estrus after calving, gestation length, insemination per conception, conception rate, daughter pregnancy rate, and pregnancy rate). These 8-traits listed here have been found to be correlated with puberty (studied traits) in cattle. For instance, inhibin is regarded as a biomarker for sexual development because it regulates spermatogenesis in both beef and dairy bulls $[53,54]$. Moderate genetic correlation was found between inhibin and AGECL [55] and between inhibin and IGF1b [56] in BB. 
355 Scrotal circumference has also been found to be a moderate predictor of AGECL and IGF1b 356 in BB [21, 26]. Thus, BTA5 may be a candidate region for fertility in BB beef cattle 357 population. Other enriched QTLs out of the 36 mentioned above include 8 different 358 production traits (average daily gain, metabolic body weight, length of productive life, body 359 weight, rump width, body depth, residual feed intake, and net merit). These traits are related 360 to feed efficiency in cattle. Improving feed efficiency of beef cattle is a major concern for 361 beef producers. A recent study from Canal, Fontes [57] found that heifers that efficiently utilize feed attain puberty early than less feed efficient ones. Moreover, heifers that attain puberty at a relatively younger age have the potential to conceive early in life and be more productive throughout their lifetime [58]. In addition, IGF1 is an effective selection tools to improve feed efficiency and other production related traits, allowing breeders to preselect animals that can utilize feed efficiently $[59,60]$. Other enriched QTLs for BTA5 in BB are related to exterior (7), milk (6), milk and carcass (5) and health (3) traits. Of note, the objective of most beef cattle breeding programs is to change the genetic merit of their cattle for many traits of interest [61]. The recurrent association of the BTA5 with multiple traits could suggest complex genetic mechanisms such as pleiotropy, epistasis, hitchhiking effects, linkage disequilibrium etc., regulating this chromosomal region [62, 63]. Therefore, breeders could target BTA5 to select multiple traits without any antagonistic effect on other traits 373 listed herein.

374 Another interesting result from this study is the shared biology between the two breeds 375 relative to the traits under study. Despite breed differences, the enriched reproductive QTLs 376 driving the genetic correlations between male and female fertility are the same for the two 377 cattle populations (Figure 6). Most of the enriched QTLs are related to reproductive traits measured early life. A possible explanation could be that the reproductive phenotypes shared common fundamental biology in the two populations. For the antagonizing regions, however, 
most of the reproductive QTLs were breed specific depending on the trait pairwise. Perhaps, this could be partly explained by the diverse genetic composition of the two breeds. Understanding the genomic architectures driving these early-in-life male and female fertility traits and their known genomic antagonisms could foster effective selection for both traits in tropical breeds $[64,65]$.

The major challenge faced by researchers when analysing an overwhelmingly large amount of genomic data is how to extract meaningful mechanistic insights into the underlying biology characterizing the given trait under study. To increase the explanatory power of genomic studies, pathway analysis has become first choice, providing researcher with the ability to infer meanings to high-throughput genomic data [66]. Leveraging the directionality of gene effects from our method with IPA knowledge base, several biological pathways known to be involved in reproduction (i.e., studied trait) were significantly enriched for all pairwise traits investigated across the two breeds. These pathways include estrogen receptor signaling, p38 MAPK signaling, GnRH signaling, sperm motility, cAMP-mediated signaling, AMPK signaling, and androgen signaling. Although IPA provided information about the activation or inhibition state for the enriched canonical metabolic pathways with the use of the $\mathbf{r}$ estimates in place of the gene expression values, we are not sure if these pathways were being activated or inhibited since we don't have information about the expression values of the genes in these pathways. For example, Rho GDP Dissociation Inhibitor (RHOGDI) pathway was the only significant signaling pathway found to be inhibited across breeds in all pairwise traits investigated using IPA comparison analysis. The RHOGDIs (RHOGDI $\alpha$, RHOGDI $\beta$ and RHOGDI $\gamma$ ) are well-characterized as a negative regulator of Rho GTPases [67]. These Rho GTPases play pivotal roles within the cell, including cell migration, membrane trafficking, invasion, gene transcription, polarity, adhesion, cell survival and death; a process significantly involved in cancer initiation and metastasis [68, 69]. Once 
405 RHOGDI is inhibited, it induces constitutive activation of Rho GTPases, resulting in several 406 malignant phenotype including tumour growth, angiogenesis, and invasive phenotypes [69,

407 70]. For instance, knocking out one of the three RHODGI genes resulted in a renal defect that 408 progressively leads to death in adult mice, although embryonic development was not affected 409 [71]. Togawa, Miyoshi [72] also found that male mice lacking RHOGDI1 were infertile with 410 impaired spermatogenesis. The authors also reported problems of implantation in female 411 mice due to this knockout. The knockout of two of the three RHOGDIs often results in a 412 more severe phenotypes with additional immunological defects than when one of the 413 RHOGDIs is disrupted [73]. Numerous studies have also reported that the RHOGDIs protein 414 are involved in sperm movement, sperm capacitation and acrosome reaction, a process that is 415 critical to occur for the sperm to interact and penetrate the egg for fertilization to take place 416 [74-76]. Perhaps, this could be the major reason why signaling by Rho family GTPases were 417 enriched in our metabolic pathway analysis. Notably, low reproduction performance is one of 418 the major challenges facing beef producers in Northern Australia [77, 78]. Reproductive 419 wastage is usually common, which is often a result of pregnancy failure and calf mortality $420[79,80]$. Given the role of the RHODGI pathway in reproduction, future studies could use 421 gene expression data to investigate the genes involved in these pathways as a candidate 422 region for infertility in cattle since we only use the $\mathbf{r}$ estimates in this study.

\section{Conclusion}

425 Overall, the framework developed in this study extends our knowledge about the regions 426 driving and antagonizing correlations between quantitative traits. About $40 \%$ of the total 427 genomic regions were identified as driving and antagonizing genetic correlations between 428 male and female fertility traits in the two population. These regions confirmed the polygenic 
nature of the traits being studied and pointed to genes of interest. Quantitative trait loci (QTL) and functional enrichment analysis revealed that many significant windows co-located with known QTLs related to milk production and fertility traits, especially puberty. In general, the enriched reproductive QTLs driving the genetic correlations between male and female

433 fertility are the same for both cattle populations, while the antagonizing regions were 434 population specific. Moreover, most of the antagonizing regions were mapped to the 435 chromosome $\mathrm{X}$. These results suggest regions of the chromosome $\mathrm{X}$ for further investigation into the trade-offs between male and female fertility. Although the methodology was applied to cattle phenotypes, using high-density SNP genotypes, the general framework developed can be applied to any species or traits, and it can easily accommodate genome sequence data.

\section{Materials and Methods}

\section{Traits, genotypes and estimated genetic correlations}

442 The traits used to demonstrate this methodology are a subset of traits from our previous study 443 [26], where bivariate genetic correlations were estimated between 7 male and 6 female early444 in-life reproductive phenotypes in two independent tropical beef cattle populations (BB and 445 TC). The two female traits selected for this study are age at detection of the first corpus 446 luteum (AGECL, days) and cows' blood concentration of insulin growth-factor 1, measured 447 at 18 months of age (IGF1c). Only one male trait was selected: the blood concentration of 448 insulin growth-factor 1, measured at 6 months of age (IGF1b). These traits are important in 449 beef cattle fertility, especially during pubertal development. The estimated genomic correlations between the traits listed above in each population have been reported in our

451 previous study [26]. These estimates and their corresponding standard error (S.E), number of 452 SNPs and number of animals in each population are provided in Table 2. These traits were 
453 selected because they had significant estimates of genomic correlation (i.e., traits with

454 standard error (S.E) less than half of the size of the correlation) and different strength or

455 direction of genetic relationships (i.e., negatively, and positively correlated traits). In brief,

456 across-sex genetic correlations were estimated in a bivariate analysis using the linear mixed

457 model approach. Firstly, the 770,000 genotypes were mapped to the new assembly of the

458 bovine reference genome (ARS_UCD1.2, GenBank assembly accession GCA_002263795.2;

459 [81]). After quality control filtering (i.e., excluding all SNPs with a minor allele frequency

460 less than 5\%), 554,712 and 686,626 SNPs remained for BB and TC datasets, respectively.

461 Finally, bivariate genetic correlations were estimated using GIBBS2F90 [82], resulting to the

462 estimates in Table 2. 
463 Table 2: Genomic correlations estimates and their corresponding standard error (s.e), 464 number of animals and number of SNPs estimates

\begin{tabular}{|c|c|c|c|}
\hline Pairwise traits & No of animals & Number of SNP & Genetic correlation (s.e) \\
\hline \multicolumn{4}{|c|}{ Brahman } \\
\hline \multirow[t]{2}{*}{ AGECL vs IGF1b } & AGECL- 980 & $554 \mathrm{~K}$ & $-0.65(0.13)$ \\
\hline & IGF1b- 964 & & \\
\hline \multirow[t]{2}{*}{ IGF1c vs IGF1b } & IGF1c- 995 & $554 \mathrm{~K}$ & $0.86(0.11)$ \\
\hline & IGF1b- 964 & & \\
\hline \multicolumn{4}{|c|}{ Tropical Composite } \\
\hline \multirow[t]{2}{*}{ AGECL vs IGF1b } & AGECL-996 & $686 \mathrm{~K}$ & $-0.55(0.14)$ \\
\hline & IGF1b- 998 & & \\
\hline IGF1b vs IGF1b & IGF1c- 1015 & $686 \mathrm{~K}$ & $0.93(0.11)$ \\
\hline
\end{tabular}

IGF1b- 998

AGECL, age at first corpus; IGF1c, serum levels of insulin growth hormone measured in

466 cow; IGF1b, serum levels of insulin growth hormone measured in bulls; SNP, Single

467 Nucleotide Polymorphisms; s.e, standard error 
469 For each trait considered in the two beef cattle populations, we estimated the genomic

470 breeding values (GEBVs) of individuals using the genomic best linear unbiased prediction

471 (GBLUP) model:

$472 \quad \mathbf{y}=\mathbf{X b}+\mathbf{Z} \mathbf{a}+\mathbf{e}$

473 where $\boldsymbol{y}$ is the vector of phenotypes, $\mathbf{X}$ is the incidence matrix of fixed effects, $\boldsymbol{b}$ is the vector

474 of fixed effects, $\mathbf{Z}$ is the design matrix assigned to genomic breeding values, $\boldsymbol{a}$ is the vector of

475 GEBVs for each animal, and $\boldsymbol{e}$ is the vector of residuals. Vectors a and $\mathbf{e}$ are assumed to

476 follow a normal distribution, thus $\mathbf{a} \sim \mathrm{N}\left(\mathbf{0}, \mathbf{G} \boldsymbol{\sigma}^{\mathbf{2}} \mathbf{g} \square\right)$ and $\mathbf{e} \sim \mathrm{N}\left(\mathbf{0}, \mathbf{I} \boldsymbol{\sigma}^{2} \mathbf{e}\right) \square$. Matrix $\mathbf{G}$ is the 477 genomic relationship matrix.

478 Following the estimation of GEBVs for each animal in each trait, we then back-solved these

479 GEBVs to obtain SNP effects for all chromosomes following the method illustrated by 480 Strandén and Garrick [83];

481

$$
\widehat{u}=\frac{1}{\sum_{j=1}^{m} 2 p_{j}(1-p)} X^{\prime} G^{-1} \widehat{a}
$$

Where $\hat{\boldsymbol{u}}$ is the vector of estimated SNP effects; $\boldsymbol{m}$ is the number of SNPs; $\boldsymbol{p}_{\boldsymbol{j}}$ is the allele frequency of the second allele of the jth marker; $\mathbf{X}$ is a matrix with gene contents for all markers; $\mathbf{G}$ is a genomic relationship matrix and $\widehat{\boldsymbol{a}}$ is a vector of GEBVs.

Notably, the estimation of GEBVs using GBLUP model [84-86] and the back-solving for GEBVs to obtain SNP effects using equation [2] both fits all SNP simultaneously, thereby allowing the joint estimation of SNP effects. As a result, the SNP effects from this approach are not biased by LD and resulting effect sizes can be considered independent.

Using a chromosome-wide approach, we divided SNPs on the same chromosome into small sliding windows of 500 SNPs each and then estimated the correlation (r) between traits as 
491 being the correlation between the 500-SNP effects estimated for trait A and the 500-SNP

492 effects estimated for trait B. We then moved 100 SNPs further from the start of the previous

493 window to select the next 500-SNP window, which partially overlapped with previous

494 window, hence producing sliding windows that were 100 SNPs distant from the previous

495 window. This was repeated for each trait pair, and for each chromosome, in a chromosome-

496 by-chromosome approach. The resulting $\mathbf{r}$ estimates for all the chromosomes combined were

497 denoted as $\mathrm{W}_{1} \ldots \mathrm{W}_{\mathrm{n}}$. The graphical illustration of this framework is presented in Figure 7.

498 Moreover, the coordinates of the windows $\left(\mathrm{W}_{1} \ldots \mathrm{W}_{\mathrm{n}}\right)$ were mapped to the ARS_UCD1.2

499 bovine reference genome. The signals across the genome were visualized with the $\mathbf{r}$ estimates

500 of each window on the y-axis and genomic position (i.e the midpoint of the start and end

501 position of each window) of each chromosome on the x-axis. The mapping to the bovine

502 reference genome and plotting of the windows signals' graphs were done using SNP \&

503 Variation Suite v8.x Golden Helix [87].

504 Depending on the overall genetic correlation observed between the traits considered, the 505 driver and antagonizing windows can be deduced. In this study, AGECL and IGF1b were 506 negatively correlated. Hence, the driver windows were windows with significant and negative $507 \mathbf{r}$ estimates, while the antagonizings were windows with significant positive $\mathbf{r}$ estimates. For 508 the positively correlated relationship between IGF1c and IGF1b, the driver windows were 509 windows with significant and positive $\mathbf{r}$ estimates and the antagonizings were windows with 510 significant and negative $\mathbf{r}$ estimates. The significance of each window was established with a 511 permutation test, described in the next section. 


\section{The graphical illustration of the framework}

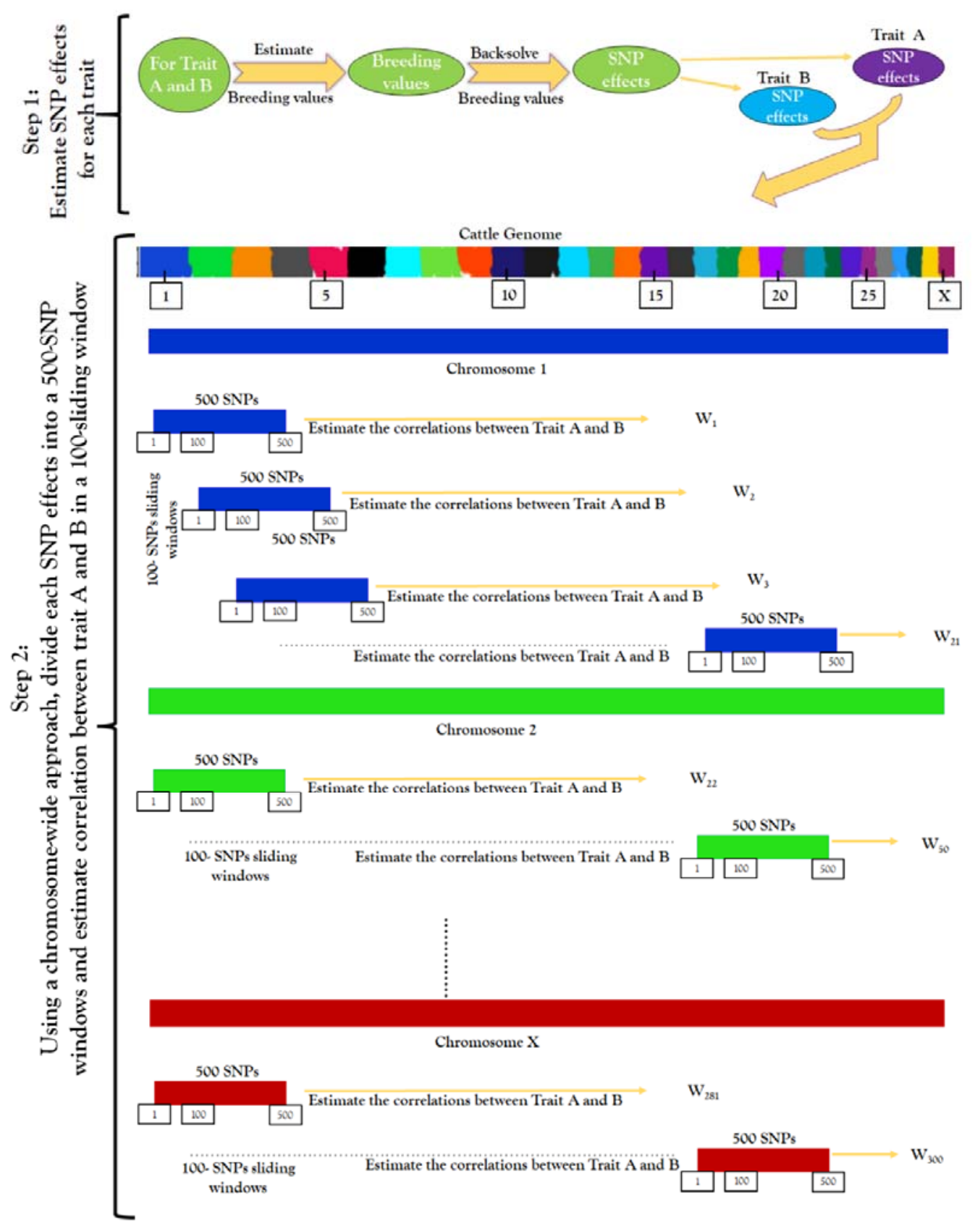

513 Figure 7. The graphical illustration of the sliding window framework. The framework 514 involves 2 steps. Step 1 start from the estimation of genomic breeding values to the 515 obtainment of SNP effects for each pairwise trait. Step 2 start from the estimation of 500516 SNP effects in a chromosome-wide approach to obtainment of the correlation estimate in a 517 100-sliding window. 


\section{Permutation test}

519 To ensure the $\mathbf{r}$ estimates are not just noise but real signals, we performed permutation test by

520 randomly reshuffling the SNP effects in each window across all the chromosomes in 1,000

521 iterations for each trait. Subsequently, we estimated correlations for 100-sliding windows of

522 500-SNP effects as described above. Finally, we observed the maximum and minimum $\mathbf{r}$

523 estimates for all the windows $\left(\mathrm{W}_{1} \ldots \ldots \mathrm{W}_{\mathrm{n}}\right)$ across the 1,000 iterations to reveal windows that

524 were significant on the pairwise traits under investigation. Afterwards, we mapped the

525 resulting $\mathbf{r}$ estimates for each window to the ARS_UCD1.2 bovine reference genome and plot

526 the $\mathbf{r}$ estimates on the $\mathrm{y}$-axis against the genomic position of each chromosome on the $\mathrm{x}$-axis

527 as described above. Consequently, significant windows were selected for the drivers and

528 antagonizings genomic regions for each pairwise. Windows that were not significant were

529 tagged "neutral windows" i.e., windows with no effect on the pairwise trait. Apart from using

530 these windows to estimate genomic correlations and investigate the proportion of variance

531 captured by these regions, they were excluded from other subsequent analyses. Finally, the $\mathbf{r}$

532 estimates of the significant windows for the driver and antagonizing regions were ranked

533 from top to bottom in percentage (\%) and the rank values were used solely for the purpose of

534 subsequent downstream analyses. The ranking was done separately for the driver and 535 antagonizing windows for each pairwise trait investigated in each population.

\section{Gene and Quantitative Traits Loci (QTL) annotation}

537 The significant windows along with their corresponding chromosome coordinates, $\mathbf{r}$

538 estimates and rank values for the driver and antagonizing regions that passed the specified

539 threshold criteria following the permutation test in BB and TC were selected. The selected

540 windows were used for gene and QTL annotation using R package GALLO: Genomic

541 Annotation in Livestock for positional candidate Loci (https://CRAN.R- 
542 project.org/package=GALLO) [27]. The .gtf annotation file corresponding to the bovine gene 543 annotation from ARS-UCD1.2 assembly and the .gff file with the QTL information from 544 cattle QTL Database (https://www.animalgenome.org/cgi-bin/QTLdb/index; [88, 89]), were 545 used for gene and QTL annotation, respectively [27]. The two files use the same bovine 546 reference genome (ARS-UCD1.2) to map the gene and QTLs. A remarkable advantage of 547 GALLO is that the software retains all the information present in the input file when 548 producing the output file. As a result, genes within each window can retain their $\mathbf{r}$ estimates 549 and the rank values specific for their window.

550 The number and percentage of genes and QTLs annotated within a population (BB or TC) 551 and the overlaps across populations (BB and TC) were investigated. Furthermore, we 552 examined the QTLs representativeness and diversity to explain better the genomic content of 553 the significant windows for the driver and antagonizing regions. Hence, the visualization of 554 the percentage of cattle QTL types from cattle QTL database (i.e milk, reproduction, 555 production, meat and carcass, health and exterior) were plotted using a pie chart by GALLO 556 (27).

\section{QTL enrichment analysis}

558 To further test the significance of the QTLs, a within population QTL enrichment analysis 559 was conducted using a chromosome-based approach. The QTL enrichment analysis, using all 560 the QTL information annotated within the significant windows for the driver and 561 antagonizing regions, was performed using the qtl_enrich function from GALLO [90, 91]. 562 Briefly, the observed number of QTLs for each trait in each annotated chromosome were 563 compared with the expected number using a hypergeometric test approach in a 1,000 iteration 564 rounds of random sampling from the entire cattle QTL database. With this approach, a p565 value for the QTL enrichment status of each annotated QTLs within the significant windows 
566 was estimated. These estimated p-values were corrected for multiple testing using a false

567 discovery rate (FDR) of $5 \%$. In addition, we used chord plots to reveal the relationships

568 between the two breeds for the enriched reproductive QTLs based on the driver and 569 antagonizing genomic regions.

\section{$570 \quad$ Functional enrichment analysis}

571 The annotated genes along with their corresponding $\mathbf{r}$ estimates and rank values for the 572 significant driver and antagonizing windows for each pairwise trait in BB and TC populations 573 were subjected to enrichment analysis using the commercial QIAGEN's Ingenuity Pathway 574 Analysis (IPA; v.8.8, http://www.ingenuity.com). The IPA allows identifying 575 overrepresented biological mechanism, metabolic pathways, and diseases and biological 576 functions that are highly relevant to the traits of interest using the directionality of the 577 submitted gene list $[92,93]$. The outcome of our methodology indicates that genes within 578 each window come with their directionalities, in this case, $r$ estimates. Thus, we leveraged on 579 the directionality of each gene by allowing the driver genes to be upregulated and 580 antagonizing genes to be downregulated.

581 Summarily, a merged dataset containing gene identifiers that were significant for both the 582 driver and antagonizing windows for each pairwise trait in each population and their 583 corresponding $\mathbf{r}$ estimates and rank values were uploaded into IPA. The $\mathbf{r}$ estimates were 584 used as the "Expr Log Ratio" and the rank values were used as p-values. The IPA software 585 recognizes gene with positive signs (+) for "Expr Log Ratio" as upregulated genes and 586 negative sign (-) as downregulated genes. We aim to allow the driver gene lists to have 587 positive values for "Expr Log Ratio" and the antagonizing gene lists to be negative. Where 588 this is not achievable based on the original $\mathbf{r}$ estimates (i.e., AGECL vs IGF1b), we reversed the sign for the driver and antagonizing genes to meet this objective. 
590 Of note, IPA can only analyse a maximum of 8,000 gene list. In most cases, the merged gene

591 list for each pairwise trait in each population is often $>8,000$. Hence, we used the rank values

592 as the cut-off to select the top $\sim 80 \%$ genes from the driver and antagonizing gene list for the

593 pathway analyses. Using a proportion of the gene list to infer biological pathways might

594 result in the loss of some important biological information relevant to the trait of interest. We

595 analysed the driver and antagonizing gene list separately for each pairwise trait in each

596 population to ensure no important information was lost because of the cut-offs. Further, we

597 compare the result of the separate analyses with the merged gene list from the $\sim 80 \%$ cut-off.

598 The pathway analysis was conducted using the "Core Analysis" function implemented within

599 IPA. In this analysis, associations were calculated using direct and indirect relationships 600 among the gene lists. At first, the gene lists were mapped to human gene data. Genes without 601 an associated gene symbol or gene annotation were subjected to an annotation by homology 602 using BioMart application available in the Ensembl database 603 (http://www.ensembl.org/biomart/martview/) [94, 95]. With this approach, we only 604 considered non-annotated genes with percentage of identity $\geq 80 \%$ with human homolog. The 605 final datasets used for the IPA analyses are presented in Additional file 8 (Table S31-34). 606 Finally, the "Core Analysis" was used to identify canonical metabolic pathways enriched at 607 Benjamini-Hochberg corrected p-values (B-H-P-value) of $\mathrm{p}<0.01)$.

\section{Supporting information}

610 Additional file 1: The number of windows, chromosome number, chromosome coordinates, 611 and correlation estimates for each window for the two pairwise trait in Brahman and Tropical 612 Composite population (Table S1-S2). 
613 Additional file 2: Genome plots of the correlation estimates from the permutation test at 500

614 iterations in $\mathrm{BB}$ and $\mathrm{TC}$ for the two pairwise traits. The correlation estimates were plotted on

615 the $y$-axis and the genomic position of each chromosome on the x-axis, according to the

616 ARS_UCD1.2 bovine reference genome (Figure S1).

617 Additional file 3: The number of windows, chromosome number, chromosome coordinates, 618 and correlation estimates as well as the maximum and minimum correlation estimate for each 619 window for all trait pairwise in Brahman and Tropical Composite population following 620 permutation test of 500-SNP effects in 100-SNP sliding windows at 1000 iterations (Table 621 S3-S6).

622 Additional file 4: The number of windows, chromosome number, chromosome coordinates, 623 correlation estimates and rank value for driver, antagonizing and the neutral regions that 624 passed the threshold after permutation test in Brahman (BB) and Tropical Composite 625 population for the studied trait (Table S7-18).

626 Additional file 5: The enriched QTLs of the driver and antagonizing regions for all trait 627 pairwise in Brahman and Tropical Composite cattle. The enriched QTLs are rank based on 628 the adj.pval (Table S19-26).

629 Additional file 6: Canonical pathways significantly enriched for all trait pairwise in Brahman 630 and Tropical Composite population. Significantly enriched canonical pathways were 631 identified using Benjamini-Hochberg p-values <0.01 (Figure S2-S9).

633 Additional file 7: The list of the significant enriched canonical metabolic pathways showing 634 all the genes involved in each pathway for all trait pairwise in Brahman and Tropical 635 Composite population. Significantly enriched canonical pathways were identified using 
636 Benjamini-Hochberg p-values $<0.01$. Z-score $>2$ denote the activation of the pathway. Z-

637 score $<-2$ indicate the inhibition of the pathway (Table S27-30).

638 Additional file 8: The final dataset used for Ingenuity Pathway Analysis (IPA) for all trait

639 pairwise in Brahman and Tropical Composite cattle (Table S31-34).

640 Abbreviations

641 AGECL: Age at first corpus luteum

642 BB: Brahman

643 BTA: Bovine chromosome

644 GBLUP: Genomic Best Linear Unbiased Prediction

645 GEBV: Genomic estimated breeding values

646 IGF1b: Serum levels of insulin growth hormone measured in bulls at 6-months of age

647 IGF1c: Serum levels of insulin growth hormone measured in bulls at 18-months of age

648 IPA: Ingenuity Pathway Analysis

649 LD: Linkage Disequilibrium

650 QTL: Quantitative Trait Loci

651 R: Correlation estimate

652 SNP: Single Nucleotide Polymorphism

653 TC: Tropical Composite

654

655 Declarations 


\section{Ethics approval and consent to participate}

657 Not applicable

\section{Consent for publication}

659 Not applicable

\section{Availability of data and materials}

661 The data used in this are available from the Cooperative Research Centre for Beef Genetic

662 Technologies (Beef CRC). Data are available from https://www.beefcrc.com with the 663 permission of Meat and Livestock Australia and the University of Queensland (if interested 664 please contact the corresponding author).

\section{Competing interests}

666 None

667 Funding

668 BSO was supported by funding from Meat and Livestock Australia (L.GEN.1710; Female 669 Fertility Phenobank and L.GEN.1818; Bull fertility update: historical data, new cohort and 670 advanced genomics) and top-up scholarship from CSIRO.

\section{Authors' contributions}

672 O.B.S and L.P.N. conceived the idea. O.B.S wrote the code, conducted the analyses, and 673 wrote the manuscript. M.S.T. and G.C.C. contributed to the analyses, manuscript writing and 674 editing. A.C. conceived the downstream biological analyses. L.R., B.J.H., and M.R.S. 675 supervised the study. All authors read, edited, and approved the final manuscript.

\section{Acknowledgements}


677 This research was conducted using the legacy database of the Cooperative Centre for Beef

678 Genetics Technologies and their core partners including Meat and Livestock Australia. The

679 authors acknowledge all the participants of the Cooperative Centre for Beef Genetics

680 Technologies for their efforts in conducting the field trials and recording the phenotypes. We

681 also acknowledge the mentorship of Professor Steve Moore to Babatunde Olasege in his PhD.

\section{References}

683 1. Liu S, Yu Y, Zhang S, Cole JB, Tenesa A, Wang T, et al. Epigenomics and genotype-phenotype

684 association analyses reveal conserved genetic architecture of complex traits in cattle and human.

685 BMC biology. 2020;18(1):1-16.

686 2. Zhang Y, Lu Q, Ye Y, Huang K, Liu W, Wu Y, et al. Local genetic correlation analysis reveals

687 heterogeneous etiologic sharing of complex traits. bioRxiv. 2020.

688 3. Cánovas A, Reverter A, DeAtley KL, Ashley RL, Colgrave ML, Fortes MR, et al. Multi-tissue

689 omics analyses reveal molecular regulatory networks for puberty in composite beef cattle. PloS one.

$690 \quad 2014 ; 9(7):$ e102551.

691 4. Fonseca PAdS, Id-Lahoucine S, Reverter A, Medrano JF, Fortes MS, Casellas J, et al.

692 Combining multi-OMICs information to identify key-regulator genes for pleiotropic effect on fertility 693 and production traits in beef cattle. PLoS One. 2018;13(10):e0205295.

$694 \quad 5 . \quad$ Lynch M, Walsh B. Genetics and analysis of quantitative traits. 1998.

695 6. Van Rheenen W, Peyrot WJ, Schork AJ, Lee SH, Wray NR. Genetic correlations of polygenic

696 disease traits: from theory to practice. Nature Reviews Genetics. 2019;20(10):567-81.

697 7. Ning Z, Pawitan Y, Shen X. High-definition likelihood inference of genetic correlations across

698 human complex traits. Nature genetics. 2020;52(8):859-64.

699 8. Mackay TF, Stone EA, Ayroles JF. The genetics of quantitative traits: challenges and

700 prospects. Nature Reviews Genetics. 2009;10(8):565-77.

7019 9. Pickrell JK, Berisa T, Liu JZ, Ségurel L, Tung JY, Hinds DA. Detection and interpretation of 702 shared genetic influences on 42 human traits. Nature genetics. 2016;48(7):709-17.

703 10. Price AL, Spencer CC, Donnelly P. Progress and promise in understanding the genetic basis of 704 common diseases. Proceedings of the Royal Society B: Biological Sciences.

705 2015;282(1821):20151684.

706 11. Lunstra D, Cundiff L. Growth and pubertal development in brahman-, boran-, tuli-, belgian

707 blue-, hereford-and angus-sired f1 bulls. Journal of Animal Science. 2003;81(6):1414-26.

708 12. Fang L, Sahana G, Ma P, Su G, Yu Y, Zhang S, et al. Use of biological priors enhances

709 understanding of genetic architecture and genomic prediction of complex traits within and between

710 dairy cattle breeds. BMC genomics. 2017;18(1):1-12.

711 13. Neyhart JL, Lorenz AJ, Smith KP. Multi-trait improvement by predicting genetic correlations

712 in breeding crosses. G3: Genes, Genomes, Genetics. 2019;9(10):3153-65.

713 14. Grotzinger AD, Rhemtulla M, de Vlaming R, Ritchie SJ, Mallard T, Hill WD, et al. Genomic

714 structural equation modelling provides insights into the multivariate genetic architecture of complex 715 traits. Nature human behaviour. 2019;3(5):513-25.

716 15. Henderson C. Recent developments in variance and covariance estimations. Journal of

717 Animal Science. 1986;63(1):208-16. 
718

719

720

721

722

723

724

725

726

727

728

729

730

731

732

733

734

735

736

737

738

739

740

741

742

743

744

745

746

747

748

749

750

751

752

753

754

755

756

757

758

759

760

761

762

763

764

16. Lee $\mathrm{SH}$, Van der Werf JH. MTG2: an efficient algorithm for multivariate linear mixed model analysis based on genomic information. Bioinformatics. 2016;32(9):1420-2.

17. Turley P, Walters RK, Maghzian O, Okbay A, Lee JJ, Fontana MA, et al. Multi-trait analysis of genome-wide association summary statistics using MTAG. Nature genetics. 2018;50(2):229-37.

18. Sodini SM, Kemper KE, Wray NR, Trzaskowski M. Comparison of genotypic and phenotypic correlations: Cheverud's conjecture in humans. Genetics. 2018;209(3):941-8.

19. Collet JM, Fuentes S, Hesketh J, Hill MS, Innocenti P, Morrow EH, et al. Rapid evolution of the intersexual genetic correlation for fitness in Drosophila melanogaster. Evolution. 2016;70(4):781-95.

20. Connallon T, Matthews G. Cross-sex genetic correlations for fitness and fitness components: connecting theoretical predictions to empirical patterns. Evolution letters. 2019;3(3):254-62.

21. Raidan FS, Porto-Neto LR, Reverter A. Across-sex genomic-assisted genetic correlations for sex-influenced traits in Brahman cattle. Genetics Selection Evolution. 2019;51(1):41.

22. Bulik-Sullivan BK, Loh P-R, Finucane HK, Ripke S, Yang J, Patterson N, et al. LD Score regression distinguishes confounding from polygenicity in genome-wide association studies. Nature genetics. 2015;47(3):291-5.

23. Shi H, Mancuso N, Spendlove S, Pasaniuc B. Local genetic correlation gives insights into the shared genetic architecture of complex traits. The American Journal of Human Genetics. 2017;101(5):737-51.

24. Werme J, van der Sluis S, Posthuma D, de Leeuw C. LAVA: An integrated framework for local genetic correlation analysis. bioRxiv. 2021:2020.12. 31.424652.

25. Zhang Y, Lu Q, Ye Y, Huang K, Liu W, Wu Y, et al. SUPERGNOVA: local genetic correlation analysis reveals heterogeneous etiologic sharing of complex traits. Genome biology. 2021;22(1):130.

26. Olasege B, Tahir M, Gouveia G, Kour J, Porto-Neto L, Hayes B, et al. Genetic parameter estimates for male and female fertility traits using genomic data to improve fertility in Australian beef cattle.

27. Fonseca PA, Suárez-Vega A, Marras G, Cánovas Á. GALLO: An R package for genomic annotation and integration of multiple data sources in livestock for positional candidate loci. GigaScience. 2020;9(12): giaa149.

28. Basso K, Margolin AA, Stolovitzky G, Klein U, Dalla-Favera R, Califano A. Reverse engineering of regulatory networks in human B cells. Nature genetics. 2005;37(4):382-90.

29. Boyle EA, Li YI, Pritchard JK. An expanded view of complex traits: from polygenic to omnigenic. Cell. 2017;169(7):1177-86.

30. Chen B-S, Yang S-K, Lan C-Y, Chuang Y-J. A systems biology approach to construct the gene regulatory network of systemic inflammation via microarray and databases mining. BMC Medical Genomics. 2008;1(1):1-22.

31. Satokangas I, Martin S, Helanterä H, Saramäki J, Kulmuni J. Multi-locus interactions and the build-up of reproductive isolation. Philosophical Transactions of the Royal Society $B$. 2020;375(1806):20190543.

32. Skelly DA, Raghupathy N, Robledo RF, Graber JH, Chesler EJ. Reference trait analysis reveals correlations between gene expression and quantitative traits in disjoint samples. Genetics. 2019;212(3):919-29.

33. Antonarakis SE, Chakravarti A, Cohen JC, Hardy J. Mendelian disorders and multifactorial traits: the big divide or one for all? Nature Reviews Genetics. 2010;11(5):380-4.

34. Goddard M, Kemper K, MacLeod I, Chamberlain A, Hayes B. Genetics of complex traits: prediction of phenotype, identification of causal polymorphisms and genetic architecture. Proceedings of the Royal Society B: Biological Sciences. 2016;283(1835):20160569. 
765

766

767

768

769

770

771

772

773

774

775

776

777

778

779

780

781

782

783

784

785

786

787

788

789

790

791

792

793

794

795

796

797

798

799

800

801

802

803

804

805

806

807

808

809

810

811

35. Moser G, Lee SH, Hayes BJ, Goddard ME, Wray NR, Visscher PM. Simultaneous discovery, estimation and prediction analysis of complex traits using a Bayesian mixture model. PLoS genetics. 2015;11(4):e1004969.

36. Fortes MR, Reverter A, Zhang Y, Collis E, Nagaraj SH, Jonsson NN, et al. Association weight matrix for the genetic dissection of puberty in beef cattle. Proceedings of the National Academy of Sciences. 2010;107(31):13642-7.

37. Bolormaa S, Hayes B, Hawken R, Zhang Y, Reverter A, Goddard M. Detection of chromosome segments of zebu and taurine origin and their effect on beef production and growth. Journal of animal science. 2011;89(7):2050-60.

38. Bolormaa S, Pryce JE, Kemper KE, Hayes BJ, Zhang Y, Tier B, et al. Detection of quantitative trait loci in Bos indicus and Bos taurus cattle using genome-wide association studies. Genetics Selection Evolution. 2013;45(1):1-12.

39. Fortes M, Kemper K, Sasazaki S, Reverter A, Pryce J, Barendse W, et al. Evidence for pleiotropism and recent selection in the PLAG 1 region in A ustralian B eef cattle. Animal genetics. 2013;44(6):636-47.

40. Juma AR, Damdimopoulou PE, Grommen SV, Van de Ven WJ, De Groef B. Emerging role of PLAG1 as a regulator of growth and reproduction. Journal of Endocrinology. 2016;228(2):R45-R56.

41. Karim L, Takeda H, Lin L, Druet T, Arias JA, Baurain D, et al. Variants modulating the expression of a chromosome domain encompassing PLAG1 influence bovine stature. Nature genetics. 2011;43(5):405-13.

42. Koufariotis L, Hayes B, Kelly M, Burns B, Lyons R, Stothard P, et al. Sequencing the mosaic genome of Brahman cattle identifies historic and recent introgression including polled. Scientific reports. 2018;8(1):1-12.

43. Littlejohn M, Grala T, Sanders K, Walker C, Waghorn G, Macdonald K, et al. Genetic variation in PLAG1 associates with early life body weight and peripubertal weight and growth in Bos taurus. Animal Genetics. 2012;43(5):591-4.

44. Nishimura S, Watanabe T, Mizoshita K, Tatsuda K, Fujita T, Watanabe N, et al. Genome-wide association study identified three major QTL for carcass weight including the PLAG1-CHCHD7 QTN for stature in Japanese Black cattle. BMC genetics. 2012;13(1):1-11.

45. Paim TdP, Hay E, Wilson C, Thomas M, Kuehn L, Paiva S, et al. Dynamics of genomic architecture during composite breed development in cattle. Animal genetics. 2020;51(2):224-34.

46. Mank JE. Population genetics of sexual conflict in the genomic era. Nature Reviews Genetics. 2017;18(12):721-30.

47. Parsch J, Ellegren $\mathrm{H}$. The evolutionary causes and consequences of sex-biased gene expression. Nature Reviews Genetics. 2013;14(2):83-7.

48. Sayadi A, Barrio AM, Immonen E, Dainat J, Berger D, Tellgren-Roth C, et al. The genomic footprint of sexual conflict. Nature ecology \& evolution. 2019;3(12):1725-30.

49. Bione S, Rizzolio F, Sala C, Ricotti R, Goegan M, Manzini M, et al. Mutation analysis of two candidate genes for premature ovarian failure, DACH2 and POF1B. Human reproduction. 2004;19(12):2759-66.

50. Jedidi I, Ouchari M, Yin Q. Sex chromosomes-linked single-gene disorders involved in human infertility. European journal of medical genetics. 2019;62(9):103560.

51. Okten G, Gunes S, Onat OE, Tukun A, Ozcelik T, Kocak I. Disruption of HDX gene in premature ovarian failure. Systems biology in reproductive medicine. 2013;59(4):218-22.

52. Weber TA, Koob S, Heide H, Wittig I, Head B, van der Bliek A, et al. APOOL is a cardiolipinbinding constituent of the Mitofilin/MINOS protein complex determining cristae morphology in mammalian mitochondria. PloS one. 2013;8(5):e63683. 
812

813

814

815

816

817

818

819

820

821

822

823

824

825

826

827

828

829

830

831

832

833

834

835

836

837

838

839

840

841

842

843

844

845

846

847

848

849

850

851

852

853

854

855

856

53. Kaneko H, Noguchi J, Kikuchi K, Akagi S, Shimada A, Taya K, et al. Production and endocrine role of inhibin during the early development of bull calves. Biology of reproduction. 2001;65(1):20915.

54. Phillips DJ. Activins, inhibins and follistatins in the large domestic species. Domestic animal endocrinology. 2005;28(1):1-16.

55. Johnston D, Corbet N, Barwick S, Wolcott ML, Holroyd R. Genetic correlations of young bull reproductive traits and heifer puberty traits with female reproductive performance in two tropical beef genotypes in northern Australia. Animal Production Science. 2014;54(1):74-84.

56. Corbet N, Burns B, Johnston D, Wolcott ML, Corbet D, Venus B, et al. Male traits and herd reproductive capability in tropical beef cattle. 2. Genetic parameters of bull traits. Animal Production Science. 2012;53(2):101-13.

57. Canal LB, Fontes PL, Sanford CD, Mercadante VR, DiLorenzo N, Lamb GC, et al. Relationships between feed efficiency and puberty in Bos taurus and Bos indicus-influenced replacement beef heifers. Journal of Animal Science. 2020;98(10):skaa319.

58. Perry GA, Cushman R. Effect of age at puberty/conception date on cow longevity. Veterinary Clinics: Food Animal Practice. 2013;29(3):579-90.

59. Bunter KL, Cai W, Johnston DJ, Dekkers JC, Bunter K. Selection to lower residual feed intake in pigs produces a correlated response in juvenile insulin-like growth factor-I (IGF-1) concentration. 60. Gao X, Xu X-R, Ren H-Y, Zhang Y-H, Xu S-Z. The effects of the GH, IGF-I and IGF-IBP3 gene on growth and development traits of Nanyang cattle in different growth period. Yi Chuan= Hereditas. 2006;28(8):927-32.

61. Bolormaa S, Pryce JE, Reverter A, Zhang Y, Barendse W, Kemper K, et al. A multi-trait, metaanalysis for detecting pleiotropic polymorphisms for stature, fatness and reproduction in beef cattle. PLoS genetics. 2014;10(3):e1004198.

62. Hackinger S, Zeggini E. Statistical methods to detect pleiotropy in human complex traits. Open biology. 2017;7(11):170125.

63. Id-Lahoucine S, Molina A, Cánovas A, Casellas J. Screening for epistatic selection signatures: A simulation study. Scientific reports. 2019;9(1):1-5.

64. Crowley J, Evans R, Mc Hugh N, Kenny D, McGee M, Crews Jr D, et al. Genetic relationships between feed efficiency in growing males and beef cow performance. Journal of animal science. 2011;89(11):3372-81.

65. Purfield DC, Evans RD, Berry DP. Breed-and trait-specific associations define the genetic architecture of calving performance traits in cattle. Journal of animal science. 2020;98(5):skaa151.

66. Nguyen T-M, Shafi A, Nguyen T, Draghici S. Identifying significantly impacted pathways: a comprehensive review and assessment. Genome biology. 2019;20(1):1-15.

67. Bozza WP, Zhang Y, Hallett K, Rosado LAR, Zhang B. RhoGDI deficiency induces constitutive activation of Rho GTPases and COX-2 pathways in association with breast cancer progression. Oncotarget. 2015;6(32):32723.

68. Hall A. Rho family gtpases. Biochemical Society Transactions. 2012;40(6):1378-82.

69. Humphries B, Wang Z, Yang C. Rho GTPases: Big Players in Breast Cancer Initiation, Metastasis and Therapeutic Responses. Cells. 2020;9(10):2167.

70. Vega FM, Ridley AJ. Rho GTPases in cancer cell biology. FEBS letters. 2008;582(14):2093-101.

71. Shibata S, Nagase M, Yoshida S, Kawarazaki W, Kurihara H, Tanaka H, et al. Modification of mineralocorticoid receptor function by Rac1 GTPase: implication in proteinuric kidney disease.

Nature medicine. 2008;14(12):1370-6. 
857 72. Togawa A, Miyoshi J, Ishizaki H, Tanaka M, Takakura A, Nishioka H, et al. Progressive

858 impairment of kidneys and reproductive organs in mice lacking Rho GDl $\alpha$. Oncogene.

859 1999;18(39):5373-80.

$86073 . \quad$ Ishizaki H, Togawa A, Tanaka-Okamoto M, Hori K, Nishimura M, Hamaguchi A, et al.

861 Defective chemokine-directed lymphocyte migration and development in the absence of Rho

862 guanosine diphosphate-dissociation inhibitors $\alpha$ and $\beta$. The Journal of Immunology.

863 2006;177(12):8512-21.

864 74. Huta Y, Nitzan Y, Breitbart H. Ezrin protects bovine spermatozoa from spontaneous

865 acrosome reaction. Theriogenology. 2020;151:119-27.

866 75. Shi Z-H, Zhao C, Wu H, Liu X-M. Expression of RhoGDI alpha in human testes and sperm and

867 its correlation with the success rate of IVF. Zhonghua nan ke xue= National Journal of Andrology.

868 2011;17(4):325-9.

869 76. Wang L, Chen W, Zhao C, Huo R, Guo X-J, Lin M, et al. The role of ezrin-associated protein

870 network in human sperm capacitation. Asian journal of andrology. 2010;12(5):667.

871 77. Burns B, Fordyce G, Holroyd R. A review of factors that impact on the capacity of beef cattle

872 females to conceive, maintain a pregnancy and wean a calf-Implications for reproductive efficiency

873 in northern Australia. Animal Reproduction Science. 2010;122(1-2):1-22.

874 78. McLean I, Holmes P, Counsell D. Final Report: The Northern beef report, 2013 Northern beef

875 siutation analysis (B. COM. 0348). Meat and Livestock Australia, Sydney. 2013.

876 79. Chang AZ, Swain DL, Trotter MG. Calf loss in northern Australia: a systematic review. The

877 Rangeland Journal. 2020;42(1):9-26.

878 80. Fordyce G. Pregnancy rates achieved by mating bulls with different percentages of

879 morphologically normal sperm. Bullpower Delivery of adequate normal sperm to site of fertilisation

880 Project NAP3. 2005;117:142-51.

881 81. Rosen BD, Bickhart DM, Schnabel RD, Koren S, Elsik CG, Tseng E, et al. De novo assembly of

882 the cattle reference genome with single-molecule sequencing. GigaScience. 2020;9(3):giaa021.

883 82. Misztal I, Tsuruta S, Strabel T, Auvray B, Druet T, Lee D, editors. BLUPF90 and related

884 programs (BGF90). Proceedings of the 7th world congress on genetics applied to livestock

885 production; 2002.

886 83. Strandén I, Garrick D. Derivation of equivalent computing algorithms for genomic predictions

887 and reliabilities of animal merit. Journal of dairy science. 2009;92(6):2971-5.

888 84. Aguilar I, Legarra A, Cardoso F, Masuda Y, Lourenco D, Misztal I. Frequentist p-values for

889 large-scale-single step genome-wide association, with an application to birth weight in American

890 Angus cattle. Genetics Selection Evolution. 2019;51(1):1-8.

891 85. VanRaden PM. Efficient methods to compute genomic predictions. Journal of dairy science.

892 2008;91(11):4414-23.

893 86. Yang J, Benyamin B, McEvoy BP, Gordon S, Henders AK, Nyholt DR, et al. Common SNPS

894 explain a large proportion of the heritability for human height. Nature genetics. 2010;42(7):565-9.

895 87. Bozeman M. Golden Helix, Inc. SNP \& Variation Suite ${ }^{\mathrm{TM}}[$ Software]. [(Version 8.x)].

896 Available from http://www.goldenhelix.com.

897 88. Hu Z-L, Park CA, Reecy JM. Developmental progress and current status of the Animal QTLdb.

898 Nucleic acids research. 2016;44(D1):D827-D33.

899 89. Hu Z-L, Park CA, Wu X-L, Reecy JM. Animal QTLdb: an improved database tool for livestock

900 animal QTL/association data dissemination in the post-genome era. Nucleic acids research.

901 2013;41(D1):D871-D9. 
902 90. Lam S, Miglior F, Fonseca P, Gómez-Redondo I, Zeidan J, Suárez-Vega A, et al. Identification

903 of functional candidate variants and genes for feed efficiency in Holstein and Jersey cattle breeds

904 using RNA-sequencing. Journal of dairy science. 2021;104(2):1928-50.

905 91. Sweett H, Fonseca P, Suarez-Vega A, Livernois A, Miglior F, Cánovas A. Genome-wide

906 association study to identify genomic regions and positional candidate genes associated with male

907 fertility in beef cattle. Scientific reports. 2020;10(1):1-14.

908 92. Krämer A, Green J, Pollard Jr J, Tugendreich S. Causal analysis approaches in ingenuity

909 pathway analysis. Bioinformatics. 2014;30(4):523-30.

910 93. Medici V, Kieffer DA, Shibata NM, Chima H, Kim K, Canovas A, et al. Wilson Disease:

911 Epigenetic effects of choline supplementation on phenotype and clinical course in a mouse model.

912 Epigenetics. 2016;11(11):804-18.

913 94. Cardoso TF, Quintanilla R, Castelló A, González-Prendes R, Amills M, Cánovas Á. Differential

914 expression of mRNA isoforms in the skeletal muscle of pigs with distinct growth and fatness profiles.

915 BMC genomics. 2018;19(1):1-12.

$91695 . \quad$ Durinck S, Moreau Y, Kasprzyk A, Davis S, De Moor B, Brazma A, et al. BioMart and

917 Bioconductor: a powerful link between biological databases and microarray data analysis.

918 Bioinformatics. 2005;21(16):3439-40.

919

920 